TRANSACTIONS OF THE

AMERICAN MATHEMATICAL SOCIETY

Volume 358, Number 10, October 2006, Pages 4225-4250

S 0002-9947(06)03960-2

Article electronically published on May 9, 2006

\title{
PALEY-WIENER THEOREMS FOR THE DUNKL TRANSFORM
}

\author{
MARCEL DE JEU
}

\begin{abstract}
We conjecture a geometrical form of the Paley-Wiener theorem for the Dunkl transform and prove three instances thereof, by using a reduction to the one-dimensional even case, shift operators, and a limit transition from Opdam's results for the graded Hecke algebra, respectively. These PaleyWiener theorems are used to extend Dunkl's intertwining operator to arbitrary smooth functions.

Furthermore, the connection between Dunkl operators and the Cartan motion group is established. It is shown how the algebra of radial parts of invariant differential operators can be described explicitly in terms of Dunkl operators. This description implies that the generalized Bessel functions coincide with the spherical functions. In this context of the Cartan motion group, the restriction of Dunkl's intertwining operator to the invariants can be interpreted in terms of the Abel transform. We also show that, for certain values of the multiplicities of the restricted roots, the Abel transform is essentially inverted by a differential operator.
\end{abstract}

\section{INTRODUCTION AND OVERVIEW}

In recent times the study of special functions associated with root systems has developed to a considerable degree. Starting with a number of conjectures by Macdonald, and the work of Heckman and Opdam on multivariable hypergeometric functions in the late 1980's, the development of the theory was greatly enhanced by the introduction of rational Dunkl operators by Dunkl [7. Through various intermediate steps of generalization, these operators can even be said to have ultimately provided crucial building blocks for Cherednik's work on double affine Hecke algebras and the $q$-Macdonald conjectures.

Originally, before the introduction of Dunkl operators, the idea when studying special functions related to root systems was to consider root multiplicities in the theory of spherical functions on Lie groups as parameters, and then to develop a theory for Weyl group invariant objects in this more general situation, without the aid of the presence of the group. It was this point of view which underlay the Macdonald conjectures and which led Heckman and Opdam to the development of their theory of hypergeometric functions in higher dimension. One of the main technical problems in this context is the description of the generalized radial parts of invariant differential operators. Apart from an explicit formula for the generalized radial part of the Laplacian - an expression which was in fact the starting point for Heckman and Opdam - the other operators remain somewhat intangible.

Received by the editors April 23, 2004.

2000 Mathematics Subject Classification. Primary 33C52; Secondary 43A32, 33C80, 22E30.

Key words and phrases. Dunkl operator, Paley-Wiener theorem, graded Hecke algebra, Cartan motion group, spherical function, multivariable Bessel function.

(C)2006 American Mathematical Society Reverts to public domain 28 years from publication 
This problem disappeared when Dunkl found the operators which have come to bear his name. They are parameterized deformations of the ordinary derivatives, involving a finite reflection group, for which it is still relatively easy to study the spectral problem and develop the theory of the corresponding Fourier transformationthe Dunkl transform. The invariant part of the theory then answers questions in a generalized theory of spherical functions - for the Cartan motion group, to be precise - as described above. The operators under consideration being explicitly given, it is actually easier to study the general context of Dunkl operators, and then specialize to invariant objects later on, than it is to restrict oneself to the invariants from the outset. The same holds true for the modification of Dunkl operators into Cherednik operators, which gives rise to a representation of the graded Hecke algebra 28] and the non-invariant "envelope" of the work of Heckman and Opdam on hypergeometric functions.

Quite remarkably, the theory of spherical functions in analysis on Lie groups can in a number of situations thus be regarded as only the invariant part of a general non-invariant theory for Dunkl-type operators. At the time of this writing, it is unknown to the author whether there is an underlying reason for this phenomenon.

In view of all this, these Dunkl operators and their modifications have attracted considerable attention in various areas of mathematics and mathematical physics during the last decade. To get an impression of their influence on the development of special functions associated with root systems - also in the general non-invariant context - we refer to, e.g., [18, 29, [16, 31. For their use in the study of integrable quantum many body systems of Calogero-Moser-Sutherland type we refer to [5] and the bibliography therein.

In this paper, we are mainly concerned with the further development of the general theory of the Dunkl transform, notably with the Paley-Wiener theorem. In addition, we describe the relation between Dunkl operators and the Cartan motion group. We will now turn to an overview of the contents of this paper with regard to these two subjects.

The first substantial results for the Dunkl transform, i.e., the Plancherel theorem and inversion theorem, were obtained by Dunkl [10] and the author 22]. Two Paley-Wiener theorems were established in [23, Chapter 3] (unpublished), of which this paper is an extension. Whereas the proof of the inversion theorem in 22 is partly a formal argument based on various symmetry properties of the eigenfunctions of Dunkl's operators, it will become apparent below that quite some more work is required in Paley-Wiener theory, due to the lack of adequate asymptotic results in the spectral domain. We will establish three Paley-Wiener theorems, each of these being a special case of a conjectured geometrically more precise general Paley-Wiener theorem, which can be found below as Conjecture 4.1, Each version requires a different technique. The first and most general version relies on a reduction to the one-dimensional even case - where asymptotic results are available - and some non-trivial results from general representation theory, and from the representation theory of the orthogonal group. The second version, which is proved for a discrete set of parameters only, is established through shift operators. Finally, the third version, which we prove for Weyl groups, follows from a limit transition from results of Opdam 28. This limit transition has some interest in itself, and for a discrete set of parameters its validity has also been established by Ben Saïd and Ørsted; cf. [2] and [3]. 
Turning to the Cartan motion group (where the material is taken from [23]), we will show how the algebra of radial parts of invariant differential operators can be described explicitly in terms of Dunkl operators with suitable values of the parameters. From this description it is easy to see that the invariant components of the eigenfunctions of the Dunkl operators are precisely the spherical functions. We can then also describe Dunkl's intertwiner operator, or rather its restriction to the invariants, in terms of the Abel transform. For certain multiplicities, the results on shift operators imply that this Abel transform is essentially inverted by a differential operator.

The organization of this paper is as follows. In Section 2 we establish the necessary general definitions and notations, and we recall some previous results. Section 3 contains a number of useful formulas. We do not claim any originality in particular for these formulas, but we believe that the proofs are considerably simpler than the ones in the existing literature. The conjectured Paley-Wiener theorem can be found in Section 4 , as can the proven three instances of it that were described above. These Paley-Wiener theorems are then used in Section 5 to extend Dunkl's intertwining operator to the smooth functions. To conclude, Section 6 contains the details of the connection between Dunkl operators and the Cartan motion group, including the results on the Abel transform.

\section{Notations AND PREvious REsults}

Let $\mathfrak{a}$ be a real vector space of finite dimension $N$ which is equipped with an inner product $(.,$.$) , inducing a Lebesgue measure d x$ on $\mathfrak{a}$. We let $\mathfrak{a}_{\mathbb{C}}=\mathfrak{a} \otimes_{\mathbb{R}} \mathbb{C}$ denote the complexification of $\mathfrak{a}$, and we extend the form $(.,$.$) to a bilinear form$ on $\mathfrak{a}_{\mathbb{C}}$, again denoted by $\left(.\right.$, . ). Both $\mathfrak{a}$ and $\mathfrak{a}_{\mathbb{C}}$ can be identified with their duals via $(.,$.$) ; for \xi$ in $\mathfrak{a}$ or $\mathfrak{a}_{\mathbb{C}}$ the corresponding linear functional is then denoted by $\xi^{*}$. Define the orthogonal group $\mathrm{O}(N)=O(\mathfrak{a},(.,)$.$) . The norm |$.$| which is induced$ on $\mathfrak{a}$ by $(.,$.$) extends to an \mathrm{O}(N)$-invariant norm on $\mathfrak{a}_{\mathbb{C}}$, also denoted by $|$.$| . There$ is a natural action of $\mathrm{O}(N)$ on functions:

$$
(g \cdot f)(x)=f\left(g^{-1} x\right) .
$$

Let $G \subset \mathrm{O}(N)$ be a finite (real) reflection group with corresponding root system $R$. We may and will assume that $(\alpha, \alpha)=2$ for all $\alpha \in R$. If $\alpha \in R$, then $r_{\alpha}$ is the orthogonal reflection in the hyperplane perpendicular to $\alpha$. We choose and fix a positive system $R_{+}$in $R$.

A function $k: R \mapsto \mathbb{C}$ is called a multiplicity function if $k$ is $G$-invariant. We write $k \geq 0$ if all values of $k$ are non-negative, with analogous notations $\operatorname{Re} k \geq 0$ and $k>0$. We let $K$ denote the vector space of multiplicity functions on $R$.

Let $k \in K$ and $\xi \in \mathfrak{a}$. Then the corresponding Dunkl operator $T_{\xi}(k)$ is defined by

$$
T_{\xi}(k)=\partial_{\xi}+\sum_{\alpha \in R_{+}} k_{\alpha}(\alpha, \xi) M_{\left(\alpha^{*}\right)^{-1}}\left(1-r_{\alpha}\right) .
$$

Here $\partial_{\xi}(\xi \in \mathfrak{a})$ is the (unnormalized) directional derivative operator; we have used the notation $M_{f}$ for pointwise multiplication by a function $f$.

The definition of the operators is independent of the choice of the positive system. The $T_{\xi}(k)$ leave the polynomials $\mathcal{P}$ invariant, mapping the homogeneous polynomials $\mathcal{P}_{n}$ of degree $n$ into $\mathcal{P}_{n-1}$. Furthermore, the spaces $C^{\infty}(\mathfrak{a})$ of smooth 
functions, $\mathcal{D}(\mathfrak{a})$ of compactly supported smooth functions and $\mathcal{S}(\mathfrak{a})$ of rapidly decreasing smooth functions are also invariant [22, Lemma 2.1].

Quite remarkably, the $T_{\xi}(k)$ form a commutative family: $T_{\xi}(k) T_{\eta}(k)=T_{\eta}(k) T_{\xi}(k)$ for all $\xi, \eta \in \mathfrak{a}$ and all $k \in K$, as was proved by Dunkl [7; see [14 for a different proof. As a consequence of the commutativity the map $\xi \mapsto T_{\xi}(k)$ extends to $\mathcal{P}$; for $p \in \mathcal{P}$ the corresponding operator is then denoted by $T_{p}(k)$. The important $k$-Laplacian $T_{|\cdot|^{2}}(k)$ is denoted by $\Delta_{k}$.

For $k \geq 0$, Dunkl $\left[8\right.$ has constructed a linear isomorphism $V_{k}: \mathcal{P} \mapsto \mathcal{P}$, homogeneous of degree 0 , such that $V_{k} 1=1$ and $T_{\xi}(k) V_{k}=V_{k} \partial_{\xi}(\xi \in \mathfrak{a})$. An alternative approach for more general $k$ can be found in [14]. A concrete description of this intertwiner operator $V_{k}$ is presently still unknown, with the exception of the onedimensional case [8] and the case $A_{2}$ [11. Significant abstract results were obtained by Rösler [30], who showed, amongst others, that $V_{k}$ is for $k \geq 0$ a positive operator which can be described in terms of measures.

Let $\lambda \in \mathfrak{a}_{\mathbb{C}}$ and consider the simultaneous eigenfunction problem

$$
T_{\xi}(k) f=(\lambda, \xi) f \quad(\xi \in \mathfrak{a}) .
$$

This problem was studied first by Dunkl [9] for $k \geq 0$; later Opdam [27] treated the general case. One more definition is needed to state the general result: a multiplicity function $k \in K$ is said to be singular if the simultaneous kernel of $\left\{T_{\xi}(k)\right\}_{\xi \in \mathfrak{a}}$ in $\mathcal{P}$ is non-trivial, i.e., if it properly contains the constants. We will use the self-evident notations $K^{\text {sing }}$ and $K^{\mathrm{reg}}$.

The set $K^{\text {sing }}$ has been determined in all cases [14, and some partial information about the simultaneous kernel for singular multiplicities can also be found in [loc. cit.]. The general nature of the simultaneous kernel for singular multiplicities is, however, still unknown, with the exception of the case $A_{n}$ of the symmetric groups which has been solved by Dunkl 12, 13. In this paper we will mainly be concerned with multiplicities satisfying $\operatorname{Re} k \geq 0$. Such multiplicities are regular, as is most easily seen by considering the operator $\sum_{i=1}^{N} e_{i}^{*} T_{e_{i}}(k)$ for an orthonormal basis $\left\{e_{1}, \ldots, e_{N}\right\}$ of $\mathfrak{a}[14$.

For regular multiplicities, the result from [27] for the eigenfunction problem is as follows.

Theorem 2.1. For all $k \in K^{\text {reg }}$ the eigenfunction problem (2.1) has a 1-dimensional solution space for all $\lambda \in \mathfrak{a}_{\mathbb{C}}$. This space contains a (unique) function $\operatorname{Exp}_{G}(\lambda, k,$. such that $\operatorname{Exp}_{G}(\lambda, k, 0)=1$. Furthermore, $\operatorname{Exp}_{G}(\lambda, k,$.$) extends to a holomorphic$ function on $\mathfrak{a}_{\mathbb{C}}$, and

$$
\operatorname{Exp}_{G}(., ., .): \mathfrak{a}_{\mathbb{C}} \mapsto \mathbb{C}
$$

is a meromorphic function with poles precisely in $K^{\text {sing }}$.

In order to be able to define the corresponding Fourier transform - the Dunkl transform - for sufficiently general functions, one needs non-trivial bounds for the eigenfunctions. It is shown in 22] that for $\operatorname{Re} k \geq 0$ one has

$$
\left|\operatorname{Exp}_{G}(\lambda, k, z)\right| \leq \sqrt{|G|} \exp \left(\max _{g \in G} \operatorname{Re}(g \lambda, z)\right) \quad\left(\lambda, z \in \mathfrak{a}_{\mathbb{C}}\right)
$$

in particular

$$
\left|\operatorname{Exp}_{G}(i \lambda, k, x)\right| \leq \sqrt{|G|} \quad(\lambda, x \in \mathfrak{a}) .
$$

If $k \geq 0$, then the constant $\sqrt{|G|}$ in (2.2) and (2.3) can in fact be improved to 1 , as a consequence of Rösler's results on the intertwiner operator [30]. 
Following Dunkl, we define for $\operatorname{Re} k \geq 0$ the $G$-invariant complex-valued weight function $w_{k}=\prod_{\alpha \in R_{+}}\left|\alpha^{*}\right|^{2 k_{\alpha}}$. The $T_{\xi}(k)$ are anti-symmetric with respect to this weight function: if $f \in \mathcal{S}(\mathfrak{a})$ and $g$ is smooth such that both $g$ and $T_{\xi}(k) g$ are of at most polynomial growth, then

$$
\int_{\mathfrak{a}}\left(T_{\xi}(k) f\right) g w_{k} d x=-\int_{\mathfrak{a}} f\left(T_{\xi}(k) g\right) w_{k} d x .
$$

In view of (2.3), the Dunkl transform for Re $k \geq 0$ is meaningfully defined on $L_{1}\left(\mathfrak{a},\left|w_{k}(x)\right| d x\right)$ as

$$
D_{k} f(\lambda)=\frac{1}{c_{k}} \int_{\mathfrak{a}} f(x) \operatorname{Exp}_{G}(-i \lambda, k, x) w_{k}(x) d x \quad\left(\lambda \in \mathfrak{a}, f \in L_{1}\left(\mathfrak{a},\left|w_{k}(x)\right| d x\right)\right) .
$$

Its alleged inverse is

$$
E_{k} f(x)=\frac{1}{c_{k}} \int_{\mathfrak{a}} f(\lambda) \operatorname{Exp}_{G}(i \lambda, k, x) w_{k}(\lambda) d \lambda \quad\left(x \in \mathfrak{a}, f \in L_{1}\left(\mathfrak{a},\left|w_{k}(\lambda)\right| d \lambda\right)\right) .
$$

Here the normalizing constant $c_{k}$ is the Mehta integral, which is defined as

$$
c_{k}=\int_{\mathfrak{a}} e^{-\frac{|x|^{2}}{2}} w_{k}(x) d x .
$$

There is a closed expression for this integral; this former Macdonald conjecture has been proved by Opdam, first for Weyl groups [26] and later for finite reflection groups in general [27]. For our needs it suffices to know that $c_{k} \neq 0$ if $\operatorname{Re} k \geq 0$, which can be proved by more elementary means [22, Corollary 4.17].

The first results for the transform, and notably a version of the Plancherel theorem, were obtained by Dunkl [10. Later on, a more systematic study was undertaken in 22]. The main properties and results are as follows.

Theorem 2.2. Let $\operatorname{Re} k \geq 0$ and $\xi \in \mathfrak{a}$. Then:

(1) $D_{k} T_{\xi}(k) f=M_{i \xi^{*}} D_{k} f(f \in \mathcal{S}(\mathfrak{a}))$.

(2) $E_{k} T_{\xi}(k) f=-M_{i \xi^{*}} E_{k} f(f \in \mathcal{S}(\mathfrak{a}))$.

(3) $D_{k} M_{i \xi^{*}} f=-T_{\xi}(k) D_{k} f(f \in \mathcal{S}(\mathfrak{a}))$.

(4) $E_{k} M_{i \xi^{*}} f=T_{\xi}(k) E_{k} f(f \in \mathcal{S}(\mathfrak{a}))$.

(5) $D_{k}$ is a linear homeomorphism of $\mathcal{S}(\mathfrak{a})$, with inverse $E_{k}$.

(6) If $f \in L_{1}\left(\mathfrak{a},\left|w_{k}(x)\right| d x\right)$ and $D_{k} f \in L_{1}\left(\mathfrak{a},\left|w_{k}(x)\right| d x\right)$, then $D_{k} E_{k} f=E_{k} D_{k} f$ $=f$ a.e.

(7) If $k \geq 0$, then $D_{k}$ maps $L_{1}\left(\mathfrak{a}, w_{k}(x) d x\right) \cap L_{2}\left(\mathfrak{a}, w_{k}(x) d x\right)$ into $L_{2}\left(\mathfrak{a}, w_{k}(x) d x\right)$, isometrically with respect to the two-norm corresponding to $w_{k}(x) d x$, and extends uniquely from $L_{1}\left(\mathfrak{a}, w_{k}(x) d x\right) \cap L_{2}\left(\mathfrak{a}, w_{k}(x) d x\right)$ to a unitary operator on $L_{2}\left(\mathfrak{a}, w_{k}(x) d x\right)$.

Remark 2.3. The definition of Dunkl operators can be generalized to complex reflection groups [15.

\section{Formularium}

In this section we establish some useful formulas. Some of them are known from the work of Dunkl, who based his proofs to a large extent on the existence of a $k$-harmonic decomposition for polynomials [6, Theorem 1.7]. However, with the 
benefit of hindsight we can simplify some of the original proofs considerably, by systematically exploiting the following commutator relation [7, Proposition 2.2]:

$$
\left[M_{\xi^{*}}, \frac{\Delta_{k}}{2}\right]=-T_{\xi}(k) \quad(\xi \in \mathfrak{a}) .
$$

To start, we first note that $\Delta_{k}: \mathcal{P} \mapsto \mathcal{P}$ is homogeneous of degree -2 , hence locally nilpotent. This enables us to define the linear automorphisms $e^{ \pm \frac{\Delta_{k}}{2}}$ of $\mathcal{P}$. These automorphisms will play an important part in what follows, together with the Gaussian $\psi(x)=\exp \left(-|x|^{2} / 2\right)$. It is known [10, 22] that the Gaussian is an eigenfunction of both $D_{k}$ and $E_{k}$ with eigenvalue 1 .

Lemma 3.1. Let $\xi \in \mathfrak{a}$. Then for arbitrary $k$ we have

(1) $\operatorname{in} \operatorname{End}_{\mathbb{C}}(\mathcal{P}):\left[M_{\xi^{*}}, e^{-\frac{\Delta_{k}}{2}}\right]=T_{\xi}(k) \circ e^{-\frac{\Delta_{k}}{2}}$;

(2) in $\operatorname{Hom}_{\mathbb{C}}(\mathcal{P}, \mathcal{S}(\mathfrak{a})): T_{\xi}(k) \circ M_{\psi} \circ e^{-\frac{\Delta_{k}}{2}}=-M_{\psi} \circ e^{-\frac{\Delta_{k}}{2}} \circ M_{\xi^{*}}$.

Proof. The first part follows immediately from (3.1) and the obvious fact that $\left[\Delta_{k}, T_{\xi}(k)\right]=0$. As to the second part, note that $T_{\xi}(k) \circ M_{\psi}=M_{\psi} \circ T_{\xi}(k)-M_{\psi} \circ$ $M_{\xi^{*}}$, as a consequence of the $G$-invariance of $\psi$. Hence

$$
\begin{aligned}
T_{\xi}(k) \circ M_{\psi} \circ e^{-\frac{\Delta_{k}}{2}} & =M_{\psi} \circ T_{\xi}(k) \circ e^{-\frac{\Delta_{k}}{2}}-M_{\psi} \circ M_{\xi^{*}} \circ e^{-\frac{\Delta_{k}}{2}} \\
& =M_{\psi} \circ T_{\xi}(k) \circ e^{-\frac{\Delta_{k}}{2}}-M_{\psi} \circ\left\{\left[M_{\xi^{*}}, e^{-\frac{\Delta_{k}}{2}}\right]+e^{-\frac{\Delta_{k}}{2}} \circ M_{\xi^{*}}\right\} \\
& =M_{\psi} \circ T_{\xi}(k) \circ e^{-\frac{\Delta_{k}}{2}}-M_{\psi} \circ\left\{T_{\xi}(k) \circ e^{-\frac{\Delta_{k}}{2}}+e^{-\frac{\Delta_{k}}{2}} \circ M_{\xi^{*}}\right\} \\
& =-M_{\psi} \circ e^{-\frac{\Delta_{k}}{2}} \circ M_{\xi^{*}} .
\end{aligned}
$$

Repeated application of the second part of Lemma 3.1 yields the formula

$$
T_{p}(k)\left\{\left(e^{-\frac{\Delta_{k}}{2}} q\right) \psi\right\}=(-1)^{\operatorname{deg} p}\left\{e^{-\frac{\Delta_{k}}{2}}(p q)\right\} \psi,
$$

for homogeneous $p \in \mathcal{P}$ and arbitrary $q \in \mathcal{P}$. Taking $q=1$ this implies, together with Theorem 2.2, the following result, which is equivalent to [10, Proposition 2.1].

Corollary 3.2. If $p \in \mathcal{P}$ is homogeneous and $\operatorname{Re} k \geq 0$, then

(1) $D_{k}(p \psi)=(-i)^{\operatorname{deg} p}\left(e^{-\frac{\Delta_{k}}{2}} p\right) \psi$.

(2) $E_{k}(p \psi)=i^{\operatorname{deg} p}\left(e^{-\frac{\Delta_{k}}{2}} p\right) \psi$.

The second part of Lemma 3.1 also enables us to reprove the symmetry of a bilinear form which was introduced by Dunkl [9], as follows. For $p, q \in \mathcal{P}$, put $(p, q)_{k}=\left(T_{p}(k) q\right)(0)$. Although it is not obvious from the definition, this bilinear form on $\mathcal{P}$ is actually symmetric. This symmetry follows from the following generalization by Dunkl [loc. cit.] of a result of Macdonald:

$$
(p, q)_{k}=\frac{1}{c_{k}} \int_{\mathfrak{a}}\left(e^{-\frac{\Delta_{k}}{2}} p\right)\left(e^{-\frac{\Delta_{k}}{2}} q\right) \psi w_{k} d x \quad(\operatorname{Re} k \geq 0, p, q \in \mathcal{P}),
$$


in which the right-hand side is obviously symmetric. In order to re-establish (3.3), denote the right-hand side by $[p, q]_{k}$. Now (2.4) and Lemma 3.1 imply that

$$
\begin{aligned}
{\left[p, T_{\xi}(k) q\right]_{k} } & =\frac{1}{c_{k}} \int_{\mathfrak{a}}\left(e^{-\frac{\Delta_{k}}{2}} p\right)\left(T_{\xi}(k) e^{-\frac{\Delta_{k}}{2}} q\right) \psi w_{k} d x \\
& =\frac{1}{c_{k}} \int_{\mathfrak{a}}-T_{\xi}(k)\left\{\left(e^{-\frac{\Delta_{k}}{2}} p\right) \psi\right\}\left(e^{-\frac{\Delta_{k}}{2}} q\right) w_{k} d x \\
& =\frac{1}{c_{k}} \int_{\mathfrak{a}}\left\{e^{-\frac{\Delta_{k}}{2}}\left(M_{\xi^{*}} p\right)\right\}\left(e^{-\frac{\Delta_{k}}{2}} q\right) \psi w_{k} d x \\
& =\left[M_{\xi^{*}} p, q\right]_{k} .
\end{aligned}
$$

But the other form $(., .)_{k}$ also has this property: $\left(M_{\xi^{*}} p, q\right)_{k}=\left(p, T_{\xi}(k) q\right)_{k}$, as a direct consequence of its definition. Since it is easy to see that $(1, q)_{k}=[1, q]_{k}$, an induction with respect to $\operatorname{deg} p$ then proves that $(p, q)_{k}=[p, q]_{k}$, which is (3.3).

The following proposition will be used in the reduction of the proof of the PaleyWiener theorem, Theorem 4.10, to the one-dimensional even case.

Proposition 3.3. If $\operatorname{Re} k$, Re $k^{\prime} \geq 0$, then

$$
E_{k^{\prime}} D_{k}(p \psi)=\left(e^{-\frac{\Delta_{k^{\prime}}}{2}} e^{\frac{\Delta_{k}}{2}} p\right) \psi \quad(p \in \mathcal{P}) .
$$

Proof. We may assume that $p$ is homogeneous. Then Corollary 3.2 implies that

$$
\begin{aligned}
E_{k^{\prime}} D_{k}(p \psi) & =(-i)^{\operatorname{deg} p} E_{k^{\prime}}\left[\left\{\sum_{n=0}^{\infty} \frac{1}{n !}\left(-\frac{\Delta_{k}}{2}\right)^{n} p\right\} \psi\right] \\
& =(-i)^{\operatorname{deg} p}\left[\sum_{n=0}^{\infty} \frac{i^{\operatorname{deg} p-2 n}}{n !} e^{-\frac{\Delta_{k^{\prime}}}{2}}\left\{\left(-\frac{\Delta_{k}}{2}\right)^{n} p\right\}\right] \psi \\
& =\left(e^{-\frac{\Delta_{k^{\prime}}}{2}} e^{\frac{\Delta_{k}}{2}} p\right) \psi .
\end{aligned}
$$

To conclude this section, we re-establish a formula of Heckman [17] which expresses the pivotal role of the $k$-Laplacian $\Delta_{k}$ :

$$
T_{p}(k)=\frac{1}{n !}\left(\operatorname{ad} \frac{\Delta_{k}}{2}\right)^{n} M_{p},
$$

for $p \in \mathcal{P}_{n}$. The proof in [loc. cit.] is based on representation theory for sl(2), but it can also be seen directly, as follows. We may assume that $M_{p}=\prod_{i=1}^{n} M_{\xi_{i}^{*}}$. Then

$$
\left(\operatorname{ad} \frac{\Delta_{k}}{2}\right)^{n} M_{p}=\sum_{\sum j_{i}=n} \frac{n !}{j_{1} ! \cdots j_{n} !}\left\{\left(\operatorname{ad} \frac{\Delta_{k}}{2}\right)^{j_{1}} M_{\xi_{1}^{*}}\right\} \circ \cdots \circ\left\{\left(\operatorname{ad} \frac{\Delta_{k}}{2}\right)^{j_{n}} M_{\xi_{n}^{*}}\right\} .
$$

But $\left(\operatorname{ad} \Delta_{k}\right)^{2} M_{\xi_{i}^{*}}=0$ as a consequence of (3.1) and the commutativity of the $T_{\xi}(k)$, so the only surviving term in the summation is the one with $j_{1}=\ldots=j_{n}=1$. Using (3.1) once more, this proves (3.4).

\section{Paley-Wiener theorems}

In this section, we conjecture a geometrical form of the Paley-Wiener theorem for the Dunkl transform, and present several theorems to support it.

Establishing notation, for $S \subset \mathfrak{a}$ we let $\mathcal{D}(S)$ denote the smooth compactly supported functions with support contained in $S$. If $S$ is compact and non-empty, then we define the indicator $I_{S}: \mathfrak{a} \mapsto \mathbb{R}$ as $I_{S}(x)=\max _{y \in S}(x, y)$ for $x \in \mathfrak{a}$. For 
such $S$, let $\mathcal{H}_{S}$ be the functions on $\mathfrak{a}_{\mathbb{C}}$ of Paley-Wiener type corresponding to $S$, i.e., those entire functions with the property that for each integer $M \geq 0$ there exists a constant $\gamma_{M}$ such that $|f(\lambda)| \leq \gamma_{M}(1+|\lambda|)^{-M} \exp I_{S}(\operatorname{Im} \lambda)$ for all $\lambda \in \mathfrak{a}_{\mathbb{C}}$. We then conjecture the following.

Conjecture 4.1 (Paley-Wiener conjecture). Let $G$ be a finite reflection group. If $\operatorname{Re} k \geq 0$ and $S$ is a non-empty $G$-invariant compact convex subset of $\mathfrak{a}$, then $D_{k}$ is a linear isomorphism between $\mathcal{D}(S)$ and $\mathcal{H}_{S}$.

There is some evidence supporting this conjecture:

- The inversion theorem and [22, Corollary 4.10] show that $D_{k}$ is an injective map from $\mathcal{D}(S)$ into $\mathcal{H}_{S}$.

- For $k=0$ the statement holds [21, Theorem 7.3.1].

- If $S$ is a ball centered at the origin, then a reduction to the one-dimensional even case, where asymptotics can be used, enables us to establish the statement as Theorem 4.10 below.

- If the $k_{\alpha}$ are all strictly positive integers, then the statement can be established, using shift operators, as Theorem 4.11 below.

- If $G$ is a Weyl group and $S$ is the intersection of convex hulls of orbits, then a limit transition from results of Opdam establishes the statement as Theorem 4.15 below.

The main obstacle for a possible proof of the conjecture along the usual lines, using a contour shift, is the absence of adequate asymptotic results for the Dunkl kernel. There are some asymptotic results available [32, but these fall far short of what is needed. It is to be expected that better results could be obtained if more was known about Rösler's representing measures [30, but as yet these remain elusive. But even if much stronger asymptotic results became available, the proof of Theorem 4.4 below seems to suggest that additional monodromy arguments may then still be necessary.

Remark 4.2. In [40, a proof of Conjecture 4.1 if $k \geq 0$ and $\sum_{\alpha \in R_{+}} k_{\alpha}>0$ is presented. That proof, however, is not correct, and to our knowledge Conjecture 4.1 is at the time of writing still open.

4.1. The case of arbitrary $G$ and $\operatorname{Re} k \geq 0$. Throughout this section, $B_{R}$ will denote the closed ball in $\mathfrak{a}$ with radius $R$ and the origin as center. The space $\mathcal{D}\left(B_{R}\right)$ carries the usual Fréchet topology of uniform convergence of all derivatives.

Our approach of the Paley-Wiener theorem for such sets - under the assumption that Re $k \geq 0$ - consists of three steps; cf. the proof of Theorem4.10. First, we prove the result for even functions in one dimension. Second, it is shown that this implies the theorem for radial functions in arbitrary dimension. In the third step we finally prove that the result for radial functions implies the theorem for general smooth functions with support in a closed ball $B_{R}$.

Remark 4.3. The special role of radial functions in the theory has been noted by several authors [23], 41], 33, 39, e.g., the Dunkl transform of a radial function is again radial [33. In Paley-Wiener theory this phenomenon is encountered once more, when reducing the radial case in arbitrary dimension to the even case in one dimension. The one-dimensional even case can be handled by either Weyl fractional integral operators 39] or by our approach below, which uses classical results for the asymptotics of Bessel functions combined with a contour shift. The final step 
in our approach, from the radial to the general case, and for which a reference to 23] was given in 39], is in a sense the deepest, since it ultimately rests on the general theory of representations of compact groups in Fréchet spaces. Recently, an alternative reduction to the one-dimensional case was introduced which circumvents the inference of the general case from the radial one [36. This reduction is based on results for $k$-harmonic polynomials, and for functions supported in $B_{R}$ it gives a proof of the Paley-Wiener theorem which is independent of the results in the present paper.

Starting with the proof, the one-dimensional even case is settled in the following theorem, where, as usual, invariance is denoted by superscripts.

Theorem 4.4. Let $\mathfrak{a}$ be one dimensional and $\operatorname{Re} k \geq 0$. If $f \in \mathcal{H}_{B_{R}}^{\mathbb{Z}_{2}}$, then $E_{k} f \in$ $\mathcal{D}\left(B_{R}\right)^{\mathbb{Z}_{2}}$.

Proof. Let $f$ be as in the statement. Then obviously $E_{k} f$ is an even rapidly decreasing smooth function. We have to show that

$$
\int_{-\infty}^{\infty} f(\lambda) \operatorname{Exp}_{\mathbb{Z}_{2}}(i \lambda, k, x)|\lambda|^{2 k} d \lambda=0
$$

if $x>R$. Fix such $x$. From [10] we have

$$
\operatorname{Exp}_{\mathbb{Z}_{2}}(i \lambda, k, x)=\Gamma\left(k+\frac{1}{2}\right)\left(\frac{\lambda x}{2}\right)^{\frac{1}{2}-k}\left\{J_{k-\frac{1}{2}}(\lambda x)+i J_{k+\frac{1}{2}}(\lambda x)\right\} .
$$

Using the invariance of $f$ we see that we are left to show that

$$
\int_{-\infty}^{\infty} f(\lambda)(\lambda x)^{\frac{1}{2}-k} J_{k-\frac{1}{2}}(\lambda x)|\lambda|^{2 k} d \lambda=0 .
$$

This expression makes it obvious that there are two obstructions for a direct application of the classical argument of shifting the contour to infinity. First, the Bessel function has exponential growth in both the positive and negative imaginary directions, and second, the weight function $|\lambda|^{2 k}$ is in general not the restriction of a holomorphic function on the upper or lower half-plane. So we proceed indirectly.

Recall the definition of the Bessel functions of the third kind:

$$
\begin{aligned}
& H_{\nu}^{(1)}=\frac{J_{-\nu}-e^{-\nu \pi i} J_{\nu}}{i \sin \nu \pi}, \\
& H_{\nu}^{(2)}=\frac{J_{-\nu}-e^{\nu \pi i} J_{\nu}}{-i \sin \nu \pi},
\end{aligned}
$$

so that

$$
J_{\nu}=\frac{H_{\nu}^{(1)}+H_{\nu}^{(2)}}{2}
$$

If $\nu$ is an integer, then a limit has to be taken. In our case, this occurs if $k$ is a half-integer, but by continuity in $k$ we may and will assume that this is not the case.

For our purposes, the important property of these functions is the asymptotic behaviour [1, 9.2.7]:

$$
H_{\nu}^{(1)}(z)=\sqrt{\frac{2}{\pi z}} e^{i\left(z-\frac{\nu \pi}{2}-\frac{\pi}{4}\right)}\left(1+O\left(\frac{1}{z}\right)\right),
$$


valid if $-\pi<\arg z<2 \pi$ (which is to be interpreted in the sense of analytic continuation). Note that the range of validity of this asymptotic development contains the entire upper half-plane and that (in contrast to the ordinary Bessel function) this Hankel function has exponential decrease in the positive imaginary direction.

Define $\phi^{(1)}, \phi^{(2)}:(0, \infty) \mapsto \mathbb{C}$ by

$$
\phi^{(1)}(\lambda)=(\lambda x)^{\frac{1}{2}-k} H_{k-\frac{1}{2}}^{(1)}(\lambda x) \lambda^{2 k}
$$

and

$$
\phi^{(2)}(\lambda)=(\lambda x)^{\frac{1}{2}-k} H_{k-\frac{1}{2}}^{(2)}(\lambda x) \lambda^{2 k} .
$$

Let $\phi_{\mathrm{c}}^{(1)}$ denote the analytic continuation of $\phi^{(1)}$ from $(0, \infty)$ to $\mathbb{C} \backslash\{i t \mid t \leq 0\}$. If one recalls that $J_{\nu}(z)=z^{\nu} \tilde{J}_{\nu}(z)$ with $\tilde{J}_{\nu}$ entire and $\mathbb{Z}_{2}$-invariant, one notes that $\phi_{\mathrm{c}}^{(1)}(\lambda)$ remains bounded as $\lambda \rightarrow 0$ in $\mathbb{C} \backslash\{i t \mid t \leq 0\}$, since $\operatorname{Re} k \geq 0$. A small computation will also make it clear that $\phi_{\mathrm{c}}^{(1)}(\lambda)=\phi^{(2)}(-\lambda)$ for $\lambda<0$.

Using the invariance of $f$ and the weight function, we then compute as follows:

$$
\begin{aligned}
\int_{-\infty}^{\infty} f(\lambda)(\lambda x)^{\frac{1}{2}-k} & J_{k-\frac{1}{2}}(\lambda x)|\lambda|^{2 k} d \lambda \\
& =2 \int_{0}^{\infty} f(\lambda)(\lambda x)^{\frac{1}{2}-k} J_{k-\frac{1}{2}}(\lambda x) \lambda^{2 k} d \lambda \\
& =\int_{0}^{\infty} f(\lambda)(\lambda x)^{\frac{1}{2}-k}\left(H_{k-\frac{1}{2}}^{(1)}(\lambda x)+H_{k-\frac{1}{2}}^{(2)}(\lambda x)\right) \lambda^{2 k} d \lambda \\
& =\int_{0}^{\infty} f(\lambda)\left(\phi^{(1)}(\lambda)+\phi^{(2)}(\lambda)\right) d \lambda \\
& =\lim _{\epsilon \downarrow 0}\left\{\int_{\epsilon}^{\infty} f(\lambda) \phi^{(1)}(\lambda) d \lambda+\int_{\epsilon}^{\infty} f(\lambda) \phi^{(2)}(\lambda) d \lambda\right\} \\
& =\lim _{\epsilon \downarrow 0}\left\{\int_{\epsilon}^{\infty} f(\lambda) \phi^{(1)}(\lambda) d \lambda+\int_{-\infty}^{-\epsilon} f(-\lambda) \phi^{(2)}(-\lambda) d \lambda\right\} \\
& =\lim _{\epsilon \downarrow 0}\left\{\int_{\epsilon}^{\infty} f(\lambda) \phi_{\mathrm{c}}^{(1)}(\lambda) d \lambda+\int_{-\infty}^{-\epsilon} f(\lambda) \phi_{\mathrm{c}}^{(1)}(\lambda) d \lambda\right\} .
\end{aligned}
$$

Now $f \phi_{\mathrm{c}}^{(1)}$ is holomorphic on $\mathbb{C} \backslash\{i t \mid t \leq 0\}$, and, since $x>R$, it has exponential decrease in the positive imaginary direction, as a consequence of (4.2). The classical argument therefore establishes (4.1), with a minor modification involving a semicircle of radius $\epsilon$ around 0 in the upper half-plane and using the fact that $f \phi_{\mathrm{c}}^{1}$ is bounded around 0 .

Next, we proceed with the reduction of the general radial case to the onedimensional even case. To this end, fix $x_{0} \neq 0$ in $\mathfrak{a}$, and define the map $\operatorname{Res}_{x_{0}}$ : $\mathcal{S}(\mathfrak{a})^{\mathrm{O}(N)} \mapsto \mathcal{S}(\mathbb{R})^{\mathbb{Z}_{2}}$ by restricting to the line passing through $x_{0}$ :

$$
\left(\operatorname{Res}_{x_{0}} f\right)(s)=f\left(s \frac{x_{0}}{\left|x_{0}\right|}\right) \quad\left(s \in \mathbb{R}, f \in \mathcal{S}(\mathfrak{a})^{\mathrm{O}(N)}\right) .
$$

The following proposition, which implies the Paley-Wiener theorem for radial functions (as will become apparent in the proof of Theorem 4.10), involves the Dunkl transform for general $\mathfrak{a}$ and $\mathbb{R}$ both at the same time. We therefore add a 
subscript $\mathbb{Z}_{2}$ in the latter case for clarity. Let $\mathcal{S}(\mathfrak{a})^{\mathrm{O}(N)}$ denote the $\mathrm{O}(N)$-invariants in $\mathcal{S}(\mathfrak{a})$.

Proposition 4.5. Suppose Re $k \geq 0$. Let $f \in \mathcal{S}(\mathfrak{a})^{\mathrm{O}(N)}$ and put $\gamma=\sum_{\alpha \in R_{+}} k_{\alpha}$. Then

$$
\operatorname{Res}_{x_{0}} E_{k} D_{0} f=E_{\gamma, \mathbb{Z}_{2}} D_{0, \mathbb{Z}_{2}} \operatorname{Res}_{x_{0}} f
$$

for all non-zero $x_{0} \in \mathfrak{a}$.

Proof. Let $\mathcal{S}_{0}(\mathfrak{a})=\{p \psi \mid p \in \mathcal{P}\}$, with $\psi$ denoting the Gaussian as in Section 3. It is known that $\mathcal{S}_{0}(\mathfrak{a})$ is dense in $\mathcal{S}(\mathfrak{a})$; see [35, p. 263] for this particular result, or [24] for a general framework for this type of problem. Note that the canonical projection from $\mathcal{S}(\mathfrak{a})$ onto $\mathcal{S}(\mathfrak{a})^{\mathrm{O}(N)}$ is continuous as a consequence of the closed graph theorem, implying that $\mathcal{S}_{0}(\mathfrak{a})^{\mathrm{O}(N)}$ is dense in $\mathcal{S}(\mathfrak{a})^{\mathrm{O}(N)}$. It is therefore, by linearity and continuity, sufficient to prove the proposition for a function $f$ of the form $|x|^{2 q} \psi$, where $q$ is a non-negative integer. This can be done using Proposition 3.3 and an identity for Laguerre polynomials. Recall the definition:

$$
L_{n}^{(\alpha)}(x)=\sum_{m=0}^{n} \frac{(-1)^{m}}{m !}\left(\begin{array}{c}
n+\alpha \\
n-m
\end{array}\right) x^{m} .
$$

Then the following identity holds [1, 22.12.6]:

$$
\sum_{m=0}^{n} L_{m}^{(\alpha)}(x) L_{n-m}^{(\beta)}(y)=L_{n}^{(\alpha+\beta+1)}(x+y) .
$$

It is known [9, Proposition 3.9] that $e^{\frac{\Delta_{0}}{2}}|x|^{2 q}=2^{q} q ! L_{q}^{(N / 2-1)}\left(-|x|^{2} / 2\right)$ and $e^{-\frac{\Delta_{k}}{2}}|x|^{2 q}=(-2)^{q} q ! L_{q}^{(N / 2+\gamma-1)}\left(|x|^{2} / 2\right)$. Using Proposition 3.3 we therefore find

$$
\begin{aligned}
E_{k} D_{0}\left(|x|^{2 q} \psi\right) & =\left(e^{-\frac{\Delta_{k}}{2}} e^{\frac{\Delta_{0}}{2}}|x|^{2 q}\right) \psi \\
& =2^{q} q ! \sum_{m=0}^{q}(-1)^{m}\left(\begin{array}{c}
N / 2+q-1 \\
q-m
\end{array}\right) L_{m}^{(N / 2+\gamma-1)}\left(|x|^{2} / 2\right) \psi \\
& =(-2)^{q} q ! \sum_{m=0}^{q}\left(\begin{array}{c}
-N / 2-m \\
q-m
\end{array}\right) L_{m}^{(N / 2+\gamma-1)}\left(|x|^{2} / 2\right) \psi
\end{aligned}
$$

The special case $n=q, y=0, \alpha=N / 2+\gamma-1$ and $\beta=-N / 2-q$ of (4.3) then shows that

$$
E_{k} D_{0}\left(|x|^{2 q} \psi\right)=(-2)^{q} q ! L_{q}^{(\gamma-q)}\left(|x|^{2} / 2\right) \psi
$$

Curiously enough, the dimension $N$ has dropped out, and the proposition follows immediately from this observation.

It follows easily from this result that the Dunkl transform of a radial function is again radial, retrieving this result from 33 .

Let $\mathcal{D}_{0}\left(B_{R}\right)=\operatorname{Span}\left\{T_{p}(0) f \mid p \in \mathcal{P}, f \in \mathcal{D}\left(B_{R}\right)^{\mathrm{O}(N)}\right\}$. The following density result is crucial in the step from the radial to the general case.

Proposition 4.6. $\mathcal{D}_{0}\left(B_{R}\right)$ is dense in $\mathcal{D}\left(B_{R}\right)$.

This proposition implies a special case of [20, Cor. 7.8, p. 310], but the latter result does not seem to imply the proposition. The proof of Proposition 4.6 is based on representation theory for compact groups in general and $\mathrm{O}(N)$ in particular; we recall a few facts to start with. 
Let $\pi: K \mapsto \operatorname{Aut}(E)$ be a strongly continuous representation of the compact group $K$ in the automorphism group of a Fréchet space $E$. A vector $e \in E$ is $K$ finite if the orbit of $e$ under $K$ spans a finite-dimensional subspace of $E$. If $\delta \in \widehat{K}$ (the unitary dual of $K$ ), then a vector $e \in E$ is $K$-finite of type $\delta$ if the span of the orbit of $e$ decomposes into finitely many copies of $\delta$. For $\delta \in \widehat{K}$, let $E_{\delta}$ denote the subspace of $K$-finite vectors of type $\delta$. By [19, Lemma 1.9, p. 396] the subspace $\bigoplus_{\delta \in \widehat{K}} E_{\delta}$ of $K$-finite vectors is dense in $E$.

The natural action of $\mathrm{O}(N)$ on $\mathcal{D}\left(B_{R}\right)$ is such a strongly continuous representation. Hence Proposition 4.6 will be implied by the density of the $K$-finite vectors, as mentioned above, once we have proved the following result.

Proposition 4.7. $\mathcal{D}_{0}\left(B_{R}\right)$ is the space of $\mathrm{O}(N)$-finite vectors in $\mathcal{D}\left(B_{R}\right)$.

Obviously, any element of $\mathcal{D}_{0}\left(B_{R}\right)$ is $\mathrm{O}(N)$-finite, but the reverse appears to be harder. The proof of Proposition 4.7 below uses some Fourier analysis; for clarity of notation, we will temporarily denote the ordinary Fourier transform by $\mathcal{F}$ rather than $D_{0}$. We need the following classical result in the representation theory of $\mathrm{O}(N)$.

Theorem 4.8. Let $\mathcal{H}_{n}$ be the harmonic polynomials in $\mathcal{P}_{n}$. Any $p \in \mathcal{P}_{n}$ has a unique decomposition of the form

$$
p=\sum_{l=0}^{\left[\frac{n}{2}\right]}|x|^{2 l} p_{l}
$$

with $p_{l} \in \mathcal{H}_{n-2 l}$. The map $\pi_{n, l}: \mathcal{P}_{n} \mapsto \mathcal{H}_{n-2 l}$ that sends $p$ to $p_{l}$ is $\mathrm{O}(N)$ equivariant. Furthermore, the representations of $\mathrm{O}(N)$ on the spaces $\mathcal{H}_{n}(n=$ $0,1, \ldots)$ are irreducible and mutually inequivalent.

As a further preparation for the proof of Proposition 4.7 we need the following result.

Lemma 4.9. Let $\delta \in \widehat{O}(N)$ and $f \in \mathcal{D}\left(B_{R}\right)$. Suppose that the orbit of $f$ under $\mathrm{O}(N)$ spans a copy $V_{\delta}$ of $\delta$ in $\mathcal{D}\left(B_{R}\right)$. Then there exist a harmonic homogeneous polynomial $p$ of type $\delta$ and scalars $\lambda_{m}(m=0,1, \ldots)$, such that $\mathcal{F}(f)(z)=$ $p(z) \sum_{m=0}^{\infty} \lambda_{m}(z, z)^{m}\left(z \in \mathbb{C}^{N}\right)$, where the series converges uniformly on compact subsets of $\mathbb{C}^{N}$.

Proof. By the Paley-Wiener theorem the Fourier transform $\mathcal{F}(f)$ is an entire function on $\mathbb{C}^{N} . \mathcal{F}$ commutes with the $\mathrm{O}(N)$-action, so there is a copy of $\delta$ in the space of functions of Paley-Wiener type. Since the action of $\mathrm{O}(N)$ on entire functions is homogeneous (in the sense that the action is on each homogeneous component of the power series development around 0 separately), we conclude that $\delta$ also occurs in $\mathcal{P}$. By Theorem 4.8 there exists a unique copy, $\mathcal{H}_{l}$ say, of $\delta$ as the harmonics of some homogeneous degree.

For $n \geq 0$, let $\pi_{n, l}$ denote the map $\mathcal{P}_{n} \mapsto \mathcal{H}_{l}$ defined by the decomposition in Theorem 4.8. Also, for $n \geq 0$ and $g \in V_{\delta}$, let $\mathcal{F}_{n}(g)$ denote the component of homogeneous degree $n$ in the power series development of $\mathcal{F}(g)$ around 0 . Consider the $\mathrm{O}(N)$-equivariant maps $\pi_{n, l} \circ \mathcal{F}_{n}: V_{\delta} \mapsto \mathcal{H}_{l}(n \geq 0)$. Since $\mathcal{F}(f) \neq 0$, there exists $n_{0}$ such that $\pi_{n_{0}, l} \circ \mathcal{F}_{n_{0}}$ is non-zero, hence an isomorphism. But then for all $n$ the map $\left(\pi_{n, l} \circ \mathcal{F}_{n}\right) \circ\left(\pi_{n_{0}, l} \circ \mathcal{F}_{n_{0}}\right)^{-1}: \mathcal{H}_{l} \mapsto \mathcal{H}_{l}$ must be equal to a multiple $\lambda_{n}$ of the identity. Hence $f(z)=\sum_{m=0}^{\infty} \lambda_{m}(z, z)^{m} p(z)\left(z \in \mathbb{C}^{N}\right)$ for some non-zero 
$p \in \mathcal{H}_{l}$. Since there exist $z \in \mathbb{C}^{N}$ with $p(z) \neq 0$ and $|z|$ arbitrarily large, the factor $p(z)$ can be brought outside the summation.

Proof of Proposition 4.7. As remarked before, $\mathcal{D}_{0}\left(B_{R}\right)$ is certainly contained in the space of $\mathrm{O}(N)$-finite vectors in $\mathcal{D}\left(B_{R}\right)$. Conversely, let $f \in \mathcal{D}\left(B_{R}\right)$ be an $\mathrm{O}(N)$ finite vector. We may assume that the orbit of $f$ spans a copy of $\delta$ in $\mathcal{D}\left(B_{R}\right)$ for some $\delta \in \widehat{O}(N)$. Applying Lemma 4.9 one obtains a factorization $\mathcal{F}(f)=p h$ of $\mathcal{F}(f)$ in a polynomial $p$ and an entire $\mathrm{O}(N)$-invariant function $h$. We proceed to show that $h$ is in fact in $\mathcal{H}_{B_{R}}$. By [34, Lemma 8.3] there exists a constant $A>0$ such that

$$
|h(z)| \leq A \int_{T^{N}}|\mathcal{F}(f)(z+w)| d \sigma_{N}(w) \quad\left(z \in \mathbb{C}^{N}\right),
$$

where $T^{N}=\left\{\left(e^{i \theta_{1}}, \ldots, e^{i \theta_{N}}\right) \mid \theta_{j} \in \mathbb{R}(j=1, \ldots, N)\right\}$ is the $N$-torus and $\sigma_{N}$ is the normalized Haar measure on $T^{N}$. There exist constants $\gamma_{M}(M=0,1,2, \ldots)$ such that

$$
|\mathcal{F}(f)(z)| \leq \gamma_{M}(1+|z|)^{-M} e^{R|\operatorname{Im} z|}\left(z \in \mathbb{C}^{N}\right)
$$

Now note that

$$
\frac{1+|z|}{1+|z+w|} \leq 1+\frac{|w|}{1+|z+w|} \leq 1+\sqrt{N} \quad\left(z \in \mathbb{C}^{N}, w \in T^{N}\right)
$$

Since in addition $|\operatorname{Im}(z+w)| \leq|\operatorname{Im} z|+\sqrt{N}$ for $z \in \mathbb{C}^{N}$ and $w \in T^{N}$, we conclude from (4.4) that $h$ also satisfies estimates as in (4.5), i.e., $h \in \mathcal{H}_{B_{R}}$. Hence $\mathcal{F}^{-1}(h)$ is in $\mathcal{D}\left(B_{R}\right)$, and in fact $\mathcal{F}^{-1} h \in \mathcal{D}\left(B_{R}\right)^{\mathrm{O}(N)}$ since $h$ is $\mathrm{O}(N)$-invariant. This implies that $f \in \mathcal{D}_{0}\left(B_{R}\right)$, as required.

This concludes the proof of Proposition 4.6.

We can now prove the Paley-Wiener theorem of this section by putting the pieces together.

Theorem 4.10 (Paley-Wiener theorem, first version). Let $G$ be a finite reflection group and suppose that $\operatorname{Re} k \geq 0$. Then the Dunkl transform $D_{k}$ is a linear isomorphism between $\mathcal{D}\left(B_{R}\right)$ and $\mathcal{H}_{B_{R}}$, for all $R \geq 0$.

Proof. In view of the inversion theorem, all that needs to be done is to show that $E_{k} g \in \mathcal{D}\left(B_{R}\right)$ for all $g \in \mathcal{H}_{B_{R}}$. To this end, start by noting that $E_{k} D_{0} f \in \mathcal{D}\left(B_{R}\right)$ if $f \in \mathcal{D}\left(B_{R}\right)^{\mathrm{O}(N)}$, as an immediate consequence of Proposition 4.5 and Theorem 4.4. But then we also have $E_{k} D_{0} f \in \mathcal{D}\left(B_{R}\right)$ for $f \in \mathcal{D}_{0}\left(B_{R}\right)$, since $E_{k} \circ D_{0} \circ \partial_{\xi}=$ $T_{\xi}(k) \circ E_{k} \circ D_{0}$. From Proposition 4.6 and continuity we can then conclude that $E_{k} D_{0} f \in \mathcal{D}\left(B_{R}\right)$ for $f \in \mathcal{D}\left(B_{R}\right)$. By the Paley-Wiener theorem for $D_{0}$, we see that $E_{k} g \in \mathcal{D}\left(B_{R}\right)$ for all $g \in \mathcal{H}_{B_{R}}$, as required.

4.2. The case of arbitrary $G$ and strictly positive integral $k$. If all $k_{\alpha}$ are strictly positive integers, Conjecture 4.1 can be established from the corresponding theorem for the Fourier transform, by showing that $\operatorname{Exp}_{G}$ is then in fact an elementary function. This can be seen by starting from Heckman's work on shift operators [17.

We introduce some notation. Let $R=\bigcup_{i=1}^{m} S_{i}$ be the decomposition of $R$ into $G$-orbits, and put $S_{i,+}=S_{i} \cap R_{+}$. Define $\pi_{i}=\prod_{\alpha \in S_{i,+}} \alpha^{*}$, and let Res denote 
restriction to the $G$-invariant functions. Then we have the following results [17, valid for $p \in \mathcal{P}^{G}$ :

$$
\operatorname{Res}\left(M_{\pi_{i}}^{-1} T_{\pi_{i}}(k)\right) \operatorname{Res}\left(T_{p}(k)\right)=\operatorname{Res}\left(T_{p}\left(k+1_{S_{i}}\right)\right) \operatorname{Res}\left(M_{\pi_{i}}^{-1} T_{\pi_{i}}(k)\right),
$$

where $\left(k+1_{S_{i}}\right)_{\alpha}=k_{\alpha}+1$ if $\alpha \in S_{i}$ and $\left(k+1_{S_{i}}\right)_{\alpha}=k_{\alpha}$ if $\alpha \notin S_{i}$.

The generalized Bessel kernel $J_{G}$ is defined for $k \in K^{\text {reg }}$ by

$$
J_{G}(\lambda, k, x)=\frac{1}{|G|} \sum_{g \in G} \operatorname{Exp}_{G}(\lambda, k, g x)
$$

and is (up to multiples) the unique $G$-invariant solution of the Bessel system

$$
T_{p}(k) f=p(\lambda) f \quad\left(p \in \mathcal{P}^{G}\right) .
$$

It follows immediately from (4.6), (4.7) and the uniqueness of invariant solutions of the Bessel system that there exist complex constants $c_{1,2}(\lambda, k)$ such that

$$
M_{\pi_{i}}^{-1} T_{\pi_{i}}(k) J_{G}(\lambda, k, .)=c_{1}(\lambda, k) J_{G}\left(\lambda, k+1_{S_{i}}, .\right)
$$

and

$$
T_{\pi_{i}}(k) M_{\pi_{i}} J_{G}\left(\lambda, k+1_{S_{i}}, .\right)=c_{2}(\lambda, k) J_{G}(\lambda, k, .) .
$$

Evaluation at zero yields $c_{2}(\lambda, k)=\left(\pi_{i}, \pi_{i}\right)_{k}$, where $(., .)_{k}$ is the bilinear form from Section 3 .

$$
\begin{aligned}
c_{1}(\lambda, k)\left(\pi_{i}, \pi_{i}\right)_{k} J_{G}(\lambda, k, .) & =c_{1}(\lambda, k) T_{\pi_{i}}(k) M_{\pi_{i}} J_{G}\left(\lambda, k+1_{S_{i}}, .\right) \\
& =T_{\pi_{i}}(k) M_{\pi_{i}} M_{\pi_{i}}^{-1} T_{\pi_{i}}(k) J_{G}(\lambda, k, .) \\
& =\pi_{i}^{2}(\lambda) J_{G}(\lambda, k, .) .
\end{aligned}
$$

Hence

$$
\pi_{i}^{2}(\lambda) J_{G}\left(\lambda, k+1_{S_{i}}, .\right)=\left(\pi_{i}, \pi_{i}\right)_{k} M_{\pi_{i}}^{-1} T_{\pi_{i}}(k) J_{G}(\lambda, k, .) .
$$

Suppose then that the (fixed) value of $k$ on $S_{i}$ is $k_{i}$, a strictly positive integer. Repeated application of (4.9) yields

$$
\begin{aligned}
w_{k}(\lambda) J_{G}(\lambda, k, .)= & \prod_{i=1}^{m} \prod_{n=1}^{k_{i}}\left(\pi_{i}, \pi_{i}\right)_{(n-1) 1_{S_{i}}+\sum_{j<i} k_{j} 1_{S_{j}}} \\
& \cdot \prod_{i=1}^{m} \prod_{n=1}^{k_{i}} M_{\pi_{i}}^{-1} T_{\pi_{i}}\left((n-1) 1_{S_{i}}+\sum_{j<i} k_{j} 1_{S_{j}}\right) \frac{1}{|G|} \sum_{g \in G} e^{(g \lambda, .)} .
\end{aligned}
$$

We conclude from (4.10) that there exist functions $p_{g}(\lambda, x)$ on $\mathfrak{a}_{\mathbb{C}} \times \mathfrak{a}_{\mathbb{C}}^{\text {reg }}$ (we suppress the dependence on our fixed $k$ ) which are polynomials in $\lambda$ with rational functions in $x$ as coefficients (with poles with respect to $x$ along the singular hyperplanes, if any) such that

$$
w_{k}(\lambda) J_{G}(\lambda, k, x)=\sum_{g \in G} p_{g}(\lambda, x) e^{(g \lambda, x)} \quad\left(\lambda \in \mathfrak{a}_{\mathbb{C}}, x \in \mathfrak{a}^{\text {reg }}\right),
$$

where as usual $\mathfrak{a}^{\text {reg }}=\{x \in \mathfrak{a} \mid(\alpha, x) \neq 0 \forall \alpha \in R\}$ and $\mathfrak{a}_{\mathbb{C}}^{\text {reg }}$ is defined similarly. Now the first operator acting in (4.10) is $T_{\pi_{1}}(0)$, showing that the $p_{g}$ are divisible 
by $\pi_{1}(\lambda)$. Since the numbering of the $S_{i}$ is arbitrary we see that the $p_{g}$ are in fact divisible by $\pi(\lambda)=\prod_{i=1}^{m} \pi_{i}(\lambda)$. Thus

$$
w_{k}(\lambda) J_{G}(\lambda, k, x)=\pi(\lambda) \sum_{g \in G} p_{g}^{\prime}(\lambda, x) e^{(g \lambda, x)} \quad\left(\lambda \in \mathfrak{a}_{\mathbb{C}}, x \in \mathfrak{a}^{\mathrm{reg}}\right),
$$

where the $p_{g}^{\prime}$ are of the same form as the $p_{g}$. It is here that we have used that the $k_{\alpha}$ are strictly positive integers, in order to obtain the factor $\pi(\lambda)$ in the right hand side of (4.11) which is needed below.

There is a similar expression for $\operatorname{Exp}_{G}$, which we obtain using a result of Opdam 27. describing how $\operatorname{Exp}_{G}$ may be obtained from $J_{G}$. According to [loc. cit., 6.4-6.6] there exists a rational function $Q(\lambda, x)$ on $\mathfrak{a}_{\mathbb{C}} \times \mathfrak{a}_{\mathbb{C}}$ such that:

- $(\lambda, x) \mapsto \pi(\lambda) Q(\lambda, x)$ is a polynomial on $\mathfrak{a}_{\mathbb{C}} \times \mathfrak{a}_{\mathbb{C}}$;

- $T_{Q(\lambda, .)}(k) J_{G}(\lambda, k,)=.\operatorname{Exp}_{G}(\lambda, k,).\left(\lambda \in \mathfrak{a}_{\mathbb{C}}^{\text {reg }}, x \in \mathfrak{a}_{\mathbb{C}}\right)$.

Using this and (4.11) we see that, for $\lambda \in \mathfrak{a}_{\mathbb{C}}^{\text {reg }}$ and $x \in \mathfrak{a}^{\text {reg }}$,

$$
\begin{aligned}
w_{k}(\lambda) \operatorname{Exp}_{G}(\lambda, k, x) & =T_{Q(\lambda, .)} w_{k}(\lambda) J_{G}(\lambda, k, .) \\
& =T_{Q(\lambda, .)} \pi(\lambda) \sum_{g \in G} p_{g}^{\prime}(\lambda, x) e^{(g \lambda, x)} \\
& =T_{\pi(\lambda) Q(\lambda, .)} \sum_{g \in G} p_{g}^{\prime}(\lambda, x) e^{(g \lambda, x)} \\
& =\sum_{g \in G} p_{g}^{\prime \prime}(\lambda, x) e^{(g \lambda, x)},
\end{aligned}
$$

where again the $p_{g}^{\prime \prime}$ are of the same form as the $p_{g}$. But then (4.12) is in fact valid by continuity for $\lambda \in \mathfrak{a}_{\mathbb{C}}$ (rather than $\lambda \in \mathfrak{a}_{\mathbb{C}}^{\text {reg }}$ ) and $x \in \mathfrak{a}^{\text {reg }}$. This observation allows us to establish Conjecture 4.1 for strictly positive integral multiplicities.

Theorem 4.11 (Paley-Wiener theorem, second version). Let $G$ be a finite reflection group and suppose that the $k_{\alpha}$ are all strictly positive integers. If $S$ is a non-empty $G$-invariant compact convex subset of $\mathfrak{a}$, then $D_{k}$ is a linear isomorphism between $\mathcal{D}(S)$ and $\mathcal{H}_{S}$.

Proof. Suppose $f \in \mathcal{H}_{S}, x \in \mathfrak{a}, x \notin S$. In view of the inversion theorem, the only thing that remains to be proved is

$$
\int_{\mathfrak{a}} f(\lambda) \operatorname{Exp}_{G}(i \lambda, k, x) w_{k}(\lambda) d \lambda=0 .
$$

If $x \in \mathfrak{a}^{\text {reg, }}$, then (4.12) allows us to write this integral as

$$
\sum_{g \in G} \int_{\mathfrak{a}} f(\lambda) p_{g}^{\prime \prime}(i \lambda, x) e^{i\left(\lambda, g^{-1} x\right)} d \lambda
$$

where the $p_{g}^{\prime \prime}$ are polynomials in $\lambda$ with rational functions in $x$ as coefficients (with poles with respect to $x$ along the singular hyperplanes, if any). Hence the geometrical form of the Paley-Wiener theorem for the Fourier transform [21, Theorem 7.3.1] shows that each of the summands is zero. Then (4.13) holds for all $x \notin S$ by continuity. 
4.3. The case where $G$ is a Weyl group and $\operatorname{Re} k \geq 0$. In the case where $G$ is a Weyl group and Re $k \geq 0$, a Paley-Wiener theorem for intersections of convex hulls of orbits can be obtained by a limit transition from the results of Opdam on Cherednik operators for $k \geq 0$ [28]. If every non-empty $G$-invariant compact convex set was the intersection of the convex hulls of orbits, then this would establish Conjecture 4.1 for Weyl groups. However, this intersection property does not hold, as the two-dimensional example of the closed unit ball and $A_{1} \times A_{1}$ shows. Thus, Conjecture 4.1 remains open even for Weyl groups, and this example also shows that in this context neither Theorem 4.10 above nor Theorem 4.15 below implies the other.

We start by collecting the relevant results from [28].

Suppose that $R$ is an integral root system, and let $k: R \mapsto \mathbb{C}$ be a multiplicity function. We will assume that $R$ is reduced, which is sufficient for our purposes. Choose a system $R_{+}$of positive roots, and define the Cherednik operator $D_{\xi}$, for $\xi \in \mathfrak{a}_{\mathbb{C}}$, as

$$
D_{\xi}(k)=\partial_{\xi}+\sum_{\alpha \in R_{+}} k_{\alpha}(\alpha, \xi) M_{\left(1-e^{\left.-\alpha^{*}\right)^{-1}}\right.}\left(1-r_{\alpha}\right)-(\rho(k), \xi),
$$

where $\rho(k)=\frac{1}{2} \sum_{\alpha \in R_{+}} k_{\alpha} \alpha$. The Cherednik operators are not equivariant, and their definition is dependent on the choice of $R_{+}$. For fixed multiplicity they form a commutative family, and together with $G$ they then generate an algebra of operators which is an isomorphic copy of the graded Hecke algebra, corresponding to the choice of $R_{+}$and $k$, as is defined by Lusztig 25. We refer to 4 for this isomorphism, or to [28]. Aside, we remark that the $D_{\xi}(k)$ certainly depend on the length of the roots, in contrast to the Dunkl operators, but it will become apparent that this is innocent for our purposes.

The Dunkl kernel $\operatorname{Exp}_{G}$ can be obtained from the eigenfunctions of the Cherednik operators by taking a suitable limit. To see this, we recall a weakened version of some of Opdam's results [28, cf. Theorem 3.15 and Proposition 6.1].

Theorem 4.12. For all $k \geq 0$ there exists an open neighborhood $U$ of $0 \in \mathfrak{a}$ and a holomorphic function $C_{G}(., k,):. \mathfrak{a}_{\mathbb{C}} \times(\mathfrak{a}+i U) \mapsto \mathbb{C}$ with the following properties:

(1) $C_{G}(\lambda, k, 0)=1$ for all $\lambda \in \mathfrak{a}_{\mathbb{C}}$;

(2) $D_{\xi}(k) C_{G}(\lambda, k,)=.(\lambda, \xi) C_{G}(\lambda, k,$.$) for all \lambda, \xi \in \mathfrak{a}_{\mathbb{C}}$;

(3) if $\lambda \in \mathfrak{a}_{\mathbb{C}}$, and $z=x+i y$ with $x \in \mathfrak{a}$ and $y \in U$, then

$$
\mid C_{G}(\lambda, k, z) \leq \sqrt{|G|} e^{-\min _{g \in G}(g \operatorname{Im} \lambda, y)+\max _{g \in G}(g \rho(k), y)+\max _{g \in G}(g \operatorname{Re} \lambda, x)} .
$$

The estimate in (4.14) enables us to prove the following limit transition.

Theorem 4.13. Let $k \geq 0$ be fixed, and suppose that $S \subset \mathfrak{a}_{\mathbb{C}} \times \mathfrak{a}_{\mathbb{C}}$ is a non-empty compact subset. Then, as $\epsilon \rightarrow 0$ through the complex numbers, we have

$$
\lim _{\epsilon \rightarrow 0} C_{G}\left(\epsilon^{-1} \lambda, k, \epsilon z\right)=\operatorname{Exp}_{G}(\lambda, k, z)
$$

uniformly for $(\lambda, z) \in S$. Here it is understood that $|\epsilon|$ is taken sufficiently small, so that $C_{G}\left(\epsilon^{-1} \lambda, k, \epsilon z\right)$ is defined for all $(\lambda, z) \in S$.

Proof. Suppose, to the contrary, that there exist $\eta>0$, a non-empty compact set $S \subset \mathfrak{a}_{\mathbb{C}} \times \mathfrak{a}_{\mathbb{C}}$, and a complex sequence $\epsilon_{n} \rightarrow 0$ such that, for all $n$,

$$
\sup _{(\lambda, z) \in S}\left|C_{G}\left(\epsilon_{n}^{-1} \lambda, k, \epsilon_{n} z\right)-\operatorname{Exp}_{G}(\lambda, k, z)\right| \geq \eta .
$$


Take an open neighborhood of $S$ of the form $V_{1} \times V_{2}$, with $V_{1}, V_{2} \subset \mathfrak{a}_{\mathbb{C}}$ open balls centered at the origin, and define $\phi_{n}: V_{1} \times V_{2} \mapsto \mathbb{C}$ by $\phi_{n}(\lambda, z)=C_{G}\left(\epsilon_{n}^{-1} \lambda, k, \epsilon_{n} z\right)$, discarding a finite number of $\epsilon_{n}$ if necessary. From (4.14) one sees that the sequence $\left\{\phi_{n}\right\}_{n=1}^{\infty}$ is uniformly bounded on $V_{1} \times V_{2}$. By Montel's theorem we may therefore assume, passing to a subsequence if necessary, that the $\phi_{n}$ converge uniformly on compact subsets of $V_{1} \times V_{2}$ to a holomorphic function $\phi_{\infty}$ on $V_{1} \times V_{2}$.

Now a small computation shows, for fixed $\lambda \in V_{1}$ and for arbitrary $\xi \in \mathfrak{a}_{\mathbb{C}}$, that as functions on $V_{2}$ we have

$$
\left\{\partial_{\xi}+\epsilon_{n} \sum_{\alpha \in R_{+}} k_{\alpha} \frac{(\alpha, \xi)}{1-e^{-\epsilon_{n}(\alpha, .)}}\left(1-r_{\alpha}\right)\right\} \phi_{n}(\lambda, .)=\left\{(\xi, \lambda)+\epsilon_{n}(\rho(k), \xi)\right\} \phi_{n}(\lambda, .) .
$$

Passing to the limit one obtains

$$
\left\{\partial_{\xi}+\sum_{\alpha \in R_{+}} k_{\alpha} \frac{(\alpha, \xi)}{(\alpha, .)}\left(1-r_{\alpha}\right)\right\} \phi_{\infty}(\lambda, .)=(\xi, \lambda) \phi_{\infty}(\lambda, .) .
$$

This is the defining equation for the Dunkl kernel, which has a local solution space spanned by $\operatorname{Exp}_{G}(\lambda, k,$.$) . Since obviously \phi_{\infty}(\lambda, 0)=1$, we conclude that $\phi_{\infty}(\lambda, z)=\operatorname{Exp}_{G}(\lambda, k, z)$ for all $(\lambda, z) \in V_{1} \times V_{2}$. But this implies that the $\phi_{n}$ converge uniformly to $\operatorname{Exp}_{G}(., k,$.$) on the compact set S \subset V_{1} \times V_{2}$ after all, which contradicts (4.16).

In the end, we will only need the special case where $\lambda \in i \mathfrak{a}$ from the following corollary. Note that the function involved is defined for all $\epsilon$ under consideration.

Corollary 4.14. Let $k \geq 0$. Then, as $\epsilon \rightarrow 0$ through the real numbers, we have $\lim _{\epsilon \rightarrow 0} C_{G}\left(\epsilon^{-1} \lambda, k, \epsilon x\right)=\operatorname{Exp}_{G}(\lambda, k, x)$ for all $\lambda \in \mathfrak{a}_{\mathbb{C}}$ and all $x \in \mathfrak{a}$.

We will now invoke Opdam's Paley-Wiener theorem. To this end, fix $x \in \mathfrak{a}$, let $\operatorname{co}(G \cdot x)$ denote the convex hull of the orbit of $x$, and fix $y \notin \operatorname{co}(G \cdot x)$. Suppose that $f \in \mathcal{H}_{\mathrm{co}(G \cdot x)}$. Then, if $k_{\alpha}>0$ for all $\alpha$, the Paley-Wiener theorem for the Cherednik transform [28, Theorem 8.6.(2)] implies that

$$
\int_{\mathfrak{a}} f(\lambda) C_{G}(i \lambda, k, y) \prod_{a \in R_{+}}\left(1-\frac{k_{\alpha}(\alpha, \alpha)}{2 i(\lambda, \alpha)}\right) \prod_{\alpha \in R_{+}}\left|\frac{\Gamma\left(\frac{2 i(\lambda, \alpha)}{(\alpha, \alpha)}+k_{\alpha}\right)}{\Gamma\left(\frac{2 i(\lambda, \alpha)}{(\alpha, \alpha)}\right)}\right|^{2} d \lambda=0,
$$

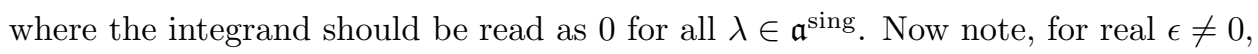
that the map $\lambda \mapsto f(\epsilon \lambda)$ is an element of $\mathcal{H}_{\operatorname{co}(G \cdot \epsilon x)}$. Since $\epsilon y \notin \operatorname{co}(G \cdot \epsilon x)$, we have

$$
\int_{\mathfrak{a}} f(\epsilon \lambda) C_{G}(i \lambda, k, \epsilon y) \prod_{a \in R_{+}}\left(1-\frac{k_{\alpha}(\alpha, \alpha)}{2 i(\lambda, \alpha)}\right) \prod_{\alpha \in R_{+}}\left|\frac{\Gamma\left(\frac{2 i(\lambda, \alpha)}{(\alpha, \alpha)}+k_{\alpha}\right)}{\Gamma\left(\frac{2 i(\lambda, \alpha)}{(\alpha, \alpha)}\right)}\right|^{2} d \lambda=0 .
$$

After a change of variables it follows that

$$
\begin{aligned}
\int_{\mathfrak{a}} f(\lambda) C_{G}\left(i \epsilon^{-1} \lambda, k, \epsilon y\right) & \prod_{a \in R_{+}}\left(1-\frac{\epsilon k_{\alpha}(\alpha, \alpha)}{2 i(\lambda, \alpha)}\right) \\
\cdot & \prod_{\alpha \in R_{+}}\left|\epsilon^{k_{\alpha}} \frac{\Gamma\left(\frac{2 i \epsilon^{-1}(\lambda, \alpha)}{(\alpha, \alpha)}+k_{\alpha}\right)}{\Gamma\left(\frac{2 i \epsilon^{-1}(\lambda, \alpha)}{(\alpha, \alpha)}\right)}\right|^{2} d \lambda=0,
\end{aligned}
$$


for all $\epsilon>0$. Now it follows from a well-known result for the Gamma function 37, p. 151] that, for each $a \in \mathbb{R}$, there exist constants $M_{1}(a), M_{2}(a), \widetilde{M}_{1}(a), \widetilde{M}_{2}(a) \geq 0$ such that, for all $s \in \mathbb{R}$,

$$
\begin{aligned}
\left|\frac{\Gamma(i s+a)}{\Gamma(i s)}\right| & \leq M_{1}(a)|s|^{a}+M_{2}(a), \\
\left|\left(1-\frac{a}{i s}\right) \frac{\Gamma(i s+a)}{\Gamma(i s)}\right| & \leq \widetilde{M}_{1}(a)|s|^{a}+\widetilde{M}_{2}(a) .
\end{aligned}
$$

Furthermore, for all $s, a \in \mathbb{R}, s \neq 0$, one has

$$
\lim _{\epsilon \downarrow 0}\left|\epsilon^{a} \frac{\Gamma\left(i \epsilon^{-1} s+a\right)}{\Gamma\left(i \epsilon^{-1} s\right)}\right|=|s|^{a} .
$$

From (4.18) and (4.19) one sees that the dominated convergence theorem applies in (4.17) as $\epsilon \downarrow 0$, since $f \in \mathcal{S}(\mathfrak{a})$ and $\left|C_{G}\left(i \epsilon^{-1} \lambda, k, \epsilon y\right)\right| \leq \sqrt{|G|}$ by (4.14). In the limit one thus obtains from (4.20) and Corollary 4.14 that

$$
\int_{\mathfrak{a}} f(\lambda) \operatorname{Exp}_{G}(i \lambda, k, y) w_{k}(\lambda) d \lambda=0,
$$

for $k>0, x \in \mathfrak{a}, f \in \mathcal{H}_{\mathrm{co}(G \cdot x)}$, and $y \notin \operatorname{co}(G \cdot x)$.

It is now an easy matter to prove the following.

Theorem 4.15 (Paley-Wiener theorem, third version). Let $G$ be a Weyl group and suppose that $\operatorname{Re} k \geq 0$. If $S \subset \mathfrak{a}$ is an intersection of convex hulls of $G$-orbits, then the Dunkl transform is a linear isomorphism between $\mathcal{D}(S)$ and $\mathcal{H}_{S}$.

Proof. As in the proof of Theorem 4.11, in view of the inversion theorem all that needs to be done is to prove that $E_{k}: \mathcal{H}_{S} \mapsto \mathcal{S}(\mathfrak{a})$ has its image contained in $\mathcal{D}(S)$. To this end, first assume that $S$ is the convex hull of one orbit and that $k>0$. In that case, (4.21) is just the required result. But then (4.21) actually holds for $\operatorname{Re} k>0$ by analytic continuation in $k$, and the case $\operatorname{Re} k \geq 0$ follows from this again by continuity. The result for an intersection of convex hulls of orbits follows trivially from the result for the convex hull of one orbit.

Remark 4.16. The type of limit transition above was introduced by Ben Saïd and Ørsted [3. The approach in [loc. cit.] starts from the invariant case, using shift operators, and then proceeds to the general case. The use of shift operators thus restricts the validity of the proofs to integral multiplicities. The approach to this transition in the present paper appears to be somewhat simpler, since Corollary 4.14 is seen to hold directly in the general case and for more general multiplicities.

\section{EXTENDING THE INTERTWINING OPERATOR}

In this section we return to the general situation of an arbitrary reflection group and $\operatorname{Re} k \geq 0$. With the aid of the Paley-Wiener theorems, we will define a linear isomorphism $V_{k}: C^{\infty}(\mathfrak{a}) \mapsto C^{\infty}(\mathfrak{a})$ such that $T_{\xi}(k) V_{k}=V_{k} \partial_{\xi}(\xi \in \mathfrak{a})$, and which extends the intertwiner operator as it was originally constructed for polynomials by Dunkl for $k \geq 0$ [8]. The polynomial case for general regular $k$ was considered in [14, and an extension of the intertwiner operator to an algebra of real analytic functions was constructed in [9] for $k \geq 0$. The extension to $C^{\infty}(\mathfrak{a})$ for $\operatorname{Re} k \geq 0$ as in this section was first established in [23]. The case where $k \geq 0$ and $\sum_{\alpha \in R_{+}} k_{\alpha}>0$ 
was later also considered in [39], using Rösler's representing measures which are presently only known to exist when $k \geq 0$.

Recall that the symmetry $S$, defined by $S f(x)=f(-x)$, relates $E_{k}$ and $D_{k}$ by $S D_{k}=D_{k} S=E_{k}$ [22, Lemma 4.3]. The Paley-Wiener theorem, Theorem 4.10, therefore implies that $D_{k} E_{0}=S E_{k} E_{0}$ maps $\mathcal{D}(\mathfrak{a})$ into itself. This map is easily seen to be continuous in the standard inductive limit topology (e.g., as a consequence of the closed graph theorem), and it is actually a homeomorphism since the inverse is $D_{0} E_{k}$. Thus the transpose $\left(D_{k} E_{0}\right)^{t}: \mathcal{D}^{\prime}(\mathfrak{a}) \mapsto \mathcal{D}^{\prime}(\mathfrak{a})$ is a homeomorphism in the weak ${ }^{*}$-topology, leaving $\mathcal{S}^{\prime}(\mathfrak{a})$ invariant as a consequence of part (5) in Theorem 2.2 .

For the formulation of the following theorem, we let $B_{R}^{\prime}$ denote the open ball of radius $R$, centered at the origin.

Theorem 5.1. Let $G$ be a finite reflection group and suppose that $\operatorname{Re} k \geq 0$. The map $W_{k}: C^{\infty}(\mathfrak{a}) \mapsto \mathcal{D}^{\prime}(\mathfrak{a})$, defined by

$$
W_{k} f=\frac{c_{0}}{c_{k}}\left(D_{k} E_{0}\right)^{t}\left(f w_{k}\right) \text {, }
$$

is actually a linear automorphism of $C^{\infty}(\mathfrak{a})$. Let $V_{k}: C^{\infty}(\mathfrak{a}) \mapsto C^{\infty}(\mathfrak{a})$ be its inverse. Then both $W_{k}$ and $V_{k}$ commute with the $G$-action, and the following hold:

(1) $W_{k} T_{\xi}(k)=\partial_{\xi} W_{k}(\xi \in \mathfrak{a})$.

(2) $W_{k} 1=1$.

(3) $W_{k} \mathcal{P}_{n}=\mathcal{P}_{n}$.

(4) (a) If $f \in C^{\infty}(\mathfrak{a})$ vanishes on $B_{R}^{\prime}$, then $W_{k} f$ vanishes on $B_{R}^{\prime}$.

(b) If $U$ is an open $G$-invariant convex subset of $\mathfrak{a}$, and $f \in C^{\infty}(\mathfrak{a})$ vanishes on $U$, then $V_{k} f$ vanishes on $U$.

(5) If $f \in C^{\infty}(\mathfrak{a}) \cap L_{1}\left(\mathfrak{a},\left|w_{k}(x)\right| d x\right), D_{k} f \in L_{1}\left(\mathfrak{a},\left|w_{k}(x)\right| d x\right)$, and $x \in \mathfrak{a}$, then

$$
W_{k} f(x)=\frac{c_{0}}{c_{k}} E_{0}\left(w_{k} D_{k} f\right)(x)=\frac{1}{c_{k}} \int_{\mathfrak{a}} D_{k} f(\lambda) \exp (i(\lambda, x)) w_{k}(\lambda) d \lambda .
$$

(6) If $f \in C^{\infty}(\mathfrak{a}) \cap L_{1}(\mathfrak{a}, d x), D_{0} f \in L_{1}(\mathfrak{a}, d x)$, and $x \in \mathfrak{a}$, then

$$
V_{k} f(x)=\frac{c_{k}}{c_{0}} E_{k}\left(w_{k}^{-1} D_{0} f\right)=\frac{1}{c_{0}} \int_{\mathfrak{a}} D_{0} f(\lambda) \operatorname{Exp}_{G}(i x, k, \lambda) d \lambda .
$$

Proof. As a preparation, we recall from [22, Lemma 4.13] that $\int_{\mathfrak{a}}\left(D_{k} f\right) g w_{k} d x=$ $\int_{\mathfrak{a}} f\left(D_{k} g\right) w_{k} d x$ for $f, g \in L_{1}\left(\mathfrak{a},\left|w_{k}(x)\right| d x\right)$, and similarly for $E_{k}$.

Suppose $T \in \mathcal{D}^{\prime}(\mathfrak{a})$ and $\operatorname{supp} T \cap B_{R}^{\prime}=\emptyset$. Then $\operatorname{supp}\left(D_{k} E_{0}\right)^{t} T \cap B_{R}^{\prime}=\emptyset$. Indeed, let $\psi \in \mathcal{D}\left(B_{R}^{\prime}\right)$. Then $\left\langle\left(D_{k} E_{0}\right)^{t} T, \psi\right\rangle=\left\langle T, D_{k} E_{0} \psi\right\rangle=\left\langle T, E_{k} D_{0} \psi\right\rangle=0$ by the Paley-Wiener theorem, Theorem 4.10 .

Now let $f \in C^{\infty}(\mathfrak{a})$. Fix $R>0$ and choose $\phi \in \mathcal{D}(\mathfrak{a})$ such that $\phi=1$ on $B_{R}^{\prime}$. Then $\operatorname{supp}\left(\phi f w_{k}-f w_{k}\right) \cap B_{R}^{\prime}=\emptyset$, hence by the above we have for arbitrary $\psi \in \mathcal{D}\left(B_{R}^{\prime}\right)$,

$$
\begin{aligned}
\left\langle\left(D_{k} E_{0}\right)^{t}\left(f w_{k}\right), \psi\right\rangle & =\left\langle\left(D_{k} E_{0}\right)^{t}\left(\phi f w_{k}\right), \psi\right\rangle \\
& =\int_{\mathfrak{a}} \psi(x) E_{0}\left(D_{k}(\phi f) w_{k}\right)(x) d x .
\end{aligned}
$$

Note that $D_{k}(\phi f) \in \mathcal{S}(\mathfrak{a})$, so $E_{0}\left(D_{k}(\phi f) w_{k}\right)$ is smooth. Since the left-hand side in (5.1) does not depend on $\phi$, the restriction of $E_{0}\left(D_{k}(\phi f) w_{k}\right)$ to $B_{R}^{\prime}$ is apparently also independent of the choice of $\phi$. Hence we can unambiguously define a smooth function representing the distribution $\left(D_{k} E_{0}\right)^{t}\left(f w_{k}\right)$, by choosing for $x \in \mathfrak{a}$ any $B_{R}^{\prime}$ such that $x \in B_{R}^{\prime}$, any $\phi \in \mathcal{D}(\mathfrak{a})$ such that $\phi=1$ on $B_{R}^{\prime}$, and putting 
$\left(\left(D_{k} E_{0}\right)^{t}\left(f w_{k}\right)\right)(x)=E_{0}\left(D_{k}(\phi f) w_{k}\right)(x)$. This shows that $W_{k}$ maps $C^{\infty}(\mathfrak{a})$ into itself. It follows from the remarks before the statement of the theorem that $W_{k}$ is injective.

In order to see that $W_{k}$ is also surjective we consider $\left(D_{0} E_{k}\right)^{t}$. A geometrically more precise property with respect to supports can be proved for $\left(D_{0} E_{k}\right)^{t}$ than for $\left(D_{k} E_{0}\right)^{t}$. To be precise, if $U$ is an open $G$-invariant convex subset of $\mathfrak{a}$, and $T \in \mathcal{D}^{\prime}(\mathfrak{a})$ is such that $\operatorname{supp} T \cap U=\emptyset$, then $\operatorname{supp}\left(D_{0} E_{k}\right)^{t} T \cap U=\emptyset$. Indeed, if $\psi \in \mathcal{D}(U)$, then $\left\langle\left(D_{0} E_{k}\right)^{t} T, \psi\right\rangle=\left\langle T, D_{0} E_{k} \psi\right\rangle=\left\langle T, E_{0} D_{k} \psi\right\rangle=0$ by the geometrical form of the Paley-Wiener theorem for the ordinary Fourier transform; note that $D_{k} \psi$ is of Paley-Wiener type corresponding to the $G$-invariant compact convex set $\operatorname{co}(G \cdot \operatorname{supp} \psi) \subset U$. Let $R>0$ and $f \in C^{\infty}(\mathfrak{a})$ and choose $\phi$ as above. Taking the open ball $B_{R}^{\prime}$ as $U$, we then compute as follows for arbitrary $\psi \in \mathcal{D}\left(B_{R}^{\prime}\right)$ :

$$
\begin{aligned}
\left\langle\left(D_{0} E_{k}\right)^{t} f, \psi\right\rangle & =\left\langle\left(D_{0} E_{k}\right)^{t}(\phi f), \psi\right\rangle \\
& =\int_{\mathfrak{a}} D_{0}(\phi f)(\lambda)\left(E_{k} \psi\right)(\lambda) d \lambda \\
& =c_{k}^{-1} \int_{\mathfrak{a}} D_{0}(\phi f)(\lambda)\left\{\int_{\mathfrak{a}} \psi(x) \operatorname{Exp}_{G}(i x, k, \lambda) w_{k}(x) d x\right\} d \lambda \\
& =\int_{\mathfrak{a}}\left\{c_{k}^{-1} \int_{\mathfrak{a}} D_{0}(\phi f)(\lambda) \operatorname{Exp}_{G}(i x, k, \lambda) d \lambda\right\} \psi(x) w_{k}(x) d x
\end{aligned}
$$

Now the function $g_{\phi}: x \mapsto c_{k}^{-1} \int_{\mathfrak{a}} D_{0}(\phi f)(\lambda) \operatorname{Exp}_{G}(i x, k, \lambda) d \lambda$ is smooth on $\mathfrak{a}$ since the derivatives of $\operatorname{Exp}_{G}(i ., k, \lambda)$ are of polynomial growth in $\lambda$ [22, Corollary 3.7] and $D_{0}(\phi f) \in \mathcal{S}(\mathfrak{a})$. The $g_{\phi}$ patch together, as before, to a smooth function $g$ on $\mathfrak{a}$ which satisfies $\left(D_{0} E_{k}\right)^{t} f=g w_{k}$ by construction. Taking inverses we find $\left(D_{k} E_{0}\right)^{t}\left(g w_{k}\right)=f$, showing that $W_{k}$ is surjective, as required.

It is easy to check that $W_{k}$ and hence its inverse $V_{k}$ commutes with the $G$-action, using an invariant function $\phi$ in the construction, and noting that $D_{k}$ and $E_{0}$ have this property.

Continuing, we define the Dunkl operator $T_{\xi}^{\prime}(k)$ on $\mathcal{D}^{\prime}(\mathfrak{a})$ by $\left\langle T_{\xi}^{\prime}(k) T, \psi\right\rangle=$ $-\left\langle T, T_{\xi}(k) \psi\right\rangle$. We have the compatibility $T_{\xi}^{\prime}(k)\left(f w_{k}\right)=\left(T_{\xi}(k) f\right) w_{k}$ for $f \in C^{\infty}(\mathfrak{a})$. Hence

$$
\begin{aligned}
\left\langle\left(D_{k} E_{0}\right)^{t}\left(\left(T_{\xi}(k) f\right) w_{k}\right), \psi\right\rangle & =\left\langle\left(T_{\xi}(k) f\right) w_{k}, D_{k} E_{0} \psi\right\rangle \\
& =\left\langle T_{\xi}^{\prime}(k)\left(f w_{k}\right), D_{k} E_{0} \psi\right\rangle \\
& =-\left\langle f w_{k}, D_{k} E_{0} \partial_{\xi} \psi\right\rangle \\
& =\left\langle\partial_{\xi}\left(\left(D_{k} E_{0}\right)^{t}\left(f w_{k}\right)\right), \psi\right\rangle,
\end{aligned}
$$

proving part (1).

For parts (2) and (3) we consider $D_{k}^{t}: \mathcal{S}^{\prime}(\mathfrak{a}) \mapsto \mathcal{S}^{\prime}(\mathfrak{a})$. By the inversion theorem we have $D_{k}^{t} w_{k}=c_{k} \delta$, hence $\left(D_{k} E_{0}\right)^{t} w_{k}=c_{k} E_{0}^{t} \delta=c_{k} c_{0}^{-1}$, proving part (2). Furthermore, one easily verifies the relation $D_{k}^{t}\left(M_{\xi^{*}} T\right)=i T_{\xi}^{\prime}(k) D_{k}^{t} T$ for $T \in \mathcal{S}^{\prime}(\mathfrak{a})$ and $\xi \in \mathfrak{a}$. Thus for $p \in \mathcal{P}_{n}$ we have $D_{k}^{t}\left(p w_{k}\right)=c_{k} i^{n} T_{p}^{\prime}(k) \delta$. By the homogeneity of the $T_{\xi}(k)$, there exists a differential operator $\widetilde{D}$ with constant coefficients and of homogeneous degree $n$ such that $c_{k} i^{n} T_{p}^{\prime}(k) \delta=\widetilde{D} \delta$. Hence $\left(D_{k} E_{0}\right)^{t}\left(p w_{k}\right)=E_{0}^{t} \widetilde{D} \delta$ is in $\mathcal{P}_{n}$, as stated in part (3). 
Part (4) follows from the properties regarding the supports of distributions which we have used above.

Parts (5) and (6) follow by performing the computations in (5.1) and (5.2) with $\phi=1$, which is validated by the integrability conditions.

Remark 5.2.

(1) It is easily seen that the validity of Conjecture 4.1 would imply that the open ball in part (4)(a) for $W_{k}$ could be replaced by an open $G$-invariant convex set, as for $V_{k}$ in part (4)(b). In fact, if all multiplicities are strictly positive integers, then we already know that this stronger statement actually holds, in view of Theorem 4.11. Likewise, if the group is a Weyl group, then we know that this stronger statement holds for the interior of an intersection of convex hulls of orbits, in view of Theorem 4.15.

(2) For $k \geq 0$, part (4)(b) also follows from the properties of Rösler's representing measures; cf. [39.

\section{Connection with the Cartan motion group}

If $G$ is a Weyl group $W$, then, for certain multiplicities, the $W$-invariant part of the theory for Dunkl operators has an interpretation in terms of the Cartan motion group, as we will explain in this section. The starting point is Heckman's result

$$
T_{p}(k)=\frac{1}{n !}\left(\operatorname{ad} \frac{\Delta_{k}}{2}\right)^{n} M_{p}
$$

for $p \in \mathcal{P}_{n}$; cf. Section 3 A double application of this result enables us to give an explicit description of the radial parts in terms of Dunkl operators. From this description we can then conclude that the generalized Bessel functions coincide with the restriction of the spherical functions to $\mathfrak{a}$. This identification, in turn, enables us to identify the restriction to the invariants of the operators $W_{k}$ and $V_{k}$ from Theorem 5.1 in terms of (the flat analogue of) the Abel transform. Furthermore, we show that in certain cases shift operators can be used to essentially invert the Abel transform by an invariant differential operator.

Establishing terminology, let $\mathcal{G}$ be a connected non-compact semisimple Lie group with finite center, maximal compact subgroup $\mathcal{K}$ and corresponding Cartan decomposition $\mathfrak{g}=\mathfrak{k} \oplus \mathfrak{p}$ of the Lie algebra. The group $\mathcal{G}_{0}=\mathcal{K} \ltimes \mathfrak{p}$ acts on the flat symmetric space $\mathcal{G}_{0} / \mathcal{K} \simeq \mathfrak{p}$ as isometries for the Killing form. The group of isometries of $\mathfrak{p}$ thus obtained is known as the Cartan motion group.

Choose a maximal abelian subspace $\mathfrak{a} \subset \mathfrak{p}$. Then there is a restriction isomorphism $\operatorname{Res}_{\mathfrak{a}}^{\mathfrak{p}}: C^{\infty}(\mathfrak{p})^{\mathcal{K}} \mapsto C^{\infty}(\mathfrak{a})^{W}$, with analogues for compactly supported smooth invariant functions and for invariant polynomials. Let $\Sigma$ be the restricted roots with multiplicities $m_{\alpha}(\alpha \in \Sigma)$. We consider $\Sigma$ to be a subset of $\mathfrak{a}$ by means of the Killing form $(.,$.$) , and we let W$ be the Weyl group, acting on $\mathfrak{a}$.

The spherical function $\psi_{\lambda}\left(\lambda \in \mathfrak{a}_{\mathbb{C}}\right)$ on the symmetric space $\mathfrak{p}$ satisfies the equations $\partial(p) \psi_{\lambda}=p(\lambda) \psi_{\lambda}$ for all $p$ in the algebra $S(\mathfrak{p})^{\mathcal{K}}$ of $\mathcal{K}$-invariant polynomials on $\mathfrak{p}$, where as usual $\partial(p)$ is the constant coefficient differential operator corresponding to $p$. Furthermore, $\psi_{\lambda}(0)=1$. By [19, Corollary 2.3, p. 402], $\psi_{\lambda}$ is the unique $\mathcal{K}$-invariant function on $\mathfrak{p}$ with these two properties.

We let $R \subset \mathfrak{a}$ be the normalized root system of the finite reflection group $W$ (so some roots in a component $B C_{n}$ of $\Sigma$ will coincide in $R$ ). Choose the multiplicity function $k: R \mapsto \mathbb{R}$ as $k_{\alpha}=1 / 4 \sum_{\beta \in \mathbb{R} \alpha \cap \Sigma} m_{\beta}$. With these multiplicities, we 
define the corresponding operators $T_{p}(k)$ (acting on functions on $\mathfrak{a}$ ) for the finite reflection group $W$. The crucial observation, with Rad denoting the radial part, is the following:

$$
\operatorname{Rad}(\partial(p)) f=T_{\operatorname{Res}_{\mathfrak{a}}^{\mathfrak{p}} p}(k) f \quad\left(p \in S(\mathfrak{p})^{\mathcal{K}}, f \in C^{\infty}(\mathfrak{a})^{W}\right) .
$$

Before proving this relation, let us note that it shows how the algebra of radial parts can be obtained from Dunkl operators, as follows. One computes the operator $T_{q}(k)$ for each polynomial $q$ in a set of fundamental invariants, and then restricts it to the invariant functions on $\mathfrak{a}$. This restriction acts as a differential operator (a moment's thought will make this clear), and the differential operators which are thus obtained generate the algebra of radial parts.

Turning to the proof of (6.2), we first recall [7] that

$$
\Delta_{k}=\Delta_{0}+2 \sum_{\alpha \in R_{+}} k_{\alpha} M_{\left(\alpha^{*}\right)^{-1}}\left\{\partial_{\alpha}-M_{\left(\alpha^{*}\right)^{-1}}\left(1-r_{\alpha}\right)\right\} .
$$

Let $\Delta_{\mathfrak{p}}$ denote the ordinary Laplacian on $\mathfrak{p}$. By the choice of $k$, the expression for $\operatorname{Rad} \Delta_{\mathfrak{p}}$ in [19, Proposition 3.13, p. 270], and the above expression for $\Delta_{k}$, we have $\operatorname{Rad} \Delta_{\mathfrak{p}} f=\Delta_{k} f$ for $f \in C^{\infty}(\mathfrak{a})^{W}$.

Now let $p \in S(\mathfrak{p})^{\mathcal{K}}$ be homogeneous of degree $n$ and suppose $f \in C^{\infty}(\mathfrak{a})^{W}$. We apply (6.1) first on $\mathfrak{p}$ (with $k=0$ ) and then on $\mathfrak{a}$ (for our particular choice of $k$ ) to prove (6.2):

$$
\begin{aligned}
\operatorname{Rad}(\partial(p)) f & =\frac{1}{n !} \operatorname{Rad}\left(\left(\operatorname{ad} \frac{\Delta_{\mathfrak{p}}}{2}\right)^{n} M_{p}\right) f \\
& =\frac{1}{n !}\left\{\left(\operatorname{ad} \frac{\operatorname{Rad} \Delta_{\mathfrak{p}}}{2}\right)^{n}\left(\operatorname{Rad} M_{p}\right)\right\} f \\
& =\frac{1}{n !}\left\{\left(\operatorname{ad} \frac{\Delta_{k}}{2}\right)^{n}\left(M_{\operatorname{Res}_{\mathfrak{a}} p}\right)\right\} f \\
& =T_{\operatorname{Res}_{\mathfrak{a}}{ }_{\mathfrak{p}}(k) f .}
\end{aligned}
$$

After having identified the radial parts in terms of Dunkl operators, it is now easy to show how the Bessel kernel $J_{W}$, i.e., the $W$-invariant component of $\operatorname{Exp}_{W}$ as in (4.8), is related to the restrictions of the spherical functions to $\mathfrak{a}$. Indeed, if $\lambda \in \mathfrak{a}_{\mathbb{C}}$, then we note that $J_{W}(\lambda, k,$.$) has an extension J_{W}^{\text {ext }}(\lambda, k,$.$) to a smooth \mathcal{K}$ invariant function on $\mathfrak{p}$. This extension is evidently equal to 1 in 0 , and satisfies the equations $\partial(p) J_{W}^{\text {ext }}(\lambda, k,)=.p(\lambda) J_{W}^{\operatorname{ext}}(\lambda, k,$.$) for all p \in S(\mathfrak{p})^{\mathcal{K}}$, as a consequence of (6.2) and the very definition of radial parts. By the uniqueness mentioned above, we conclude that $J_{W}^{\operatorname{ext}}(\lambda, k, x)=\psi_{\lambda}(x)$ for $\lambda \in \mathfrak{a}_{\mathbb{C}}$ and $x \in \mathfrak{a}$.

In this context of the Cartan motion group, we will now proceed to identify the restrictions to the invariants $C^{\infty}(\mathfrak{a})^{W}$ of the linear automorphisms $W_{k}, V_{k}$ : $C^{\infty}(\mathfrak{a}) \mapsto C^{\infty}(\mathfrak{a})$ from Theorem 5.1, Recall [19, p. 467] that

$$
\int_{\mathfrak{p}} f(x) d x=(2 \pi)^{\frac{\operatorname{dim} \mathfrak{p}}{2}} c_{k}^{-1} \int_{\mathfrak{a}} \operatorname{Res}_{\mathfrak{a}}^{\mathfrak{p}} f(x) w_{k} d x \quad\left(f \in C_{c}^{\infty}(\mathfrak{p})^{\mathcal{K}}\right) .
$$

The constant is determined by considering the Gaussian. Since we have identified the spherical functions as Bessel functions, we see from this integral formula that the normalized spherical transform $\mathcal{F}$, defined by

$$
\mathcal{F} f(\lambda)=(2 \pi)^{-\frac{\operatorname{dim} \mathfrak{p}}{2}} \int_{\mathfrak{p}} f(x) \psi_{-i \lambda}(x) d x \quad\left(\lambda \in \mathfrak{a}, f \in C_{c}^{\infty}(\mathfrak{p})^{\mathcal{K}}\right),
$$


factors as $\mathcal{F}=D_{k} \operatorname{Res}_{\mathfrak{a}}^{\mathfrak{p}}$. From this relation one sees that the inversion, Plancherel and Paley-Wiener theorems for the spherical transform follow from the corresponding theorems for $D_{k}$, when specialized to the invariants (the first two admittedly being almost trivial for the Cartan motion group).

Let $\mathfrak{q}$ be the orthocomplement of $\mathfrak{a}$ in $\mathfrak{p}$ and define the (flat analogue of the) Abel transform:

$$
A f(x)=\int_{\mathfrak{q}} f(x+q) d q \quad\left(f \in C_{c}^{\infty}(\mathfrak{p})^{\mathcal{K}}, x \in \mathfrak{a}\right) .
$$

Since the spherical transform can also be written as

$$
\mathcal{F} f(\lambda)=(2 \pi)^{-\frac{\mathrm{dim} \mathfrak{p}}{2}} \int_{\mathfrak{p}} f(x) e^{-i(\lambda, x)} d x \quad\left(\lambda \in \mathfrak{a}, f \in C_{c}^{\infty}(\mathfrak{p})^{\mathcal{K}}\right),
$$

Fubini's theorem yields an additional factorization of $\mathcal{F}$, namely $\mathcal{F}=(2 \pi)^{-\frac{\text { dim } \mathfrak{q}}{2}} D_{0} A$. The relation $(2 \pi)^{-\frac{\operatorname{dim} \mathfrak{q}}{2}} D_{0} A f=D_{k} \operatorname{Res}_{\mathfrak{a}}^{\mathfrak{p}} f$ for $f \in C_{c}^{\infty}(\mathfrak{p})^{\mathcal{K}}$ together with the PaleyWiener theorems for $D_{0}$ and $D_{k}$ then show that the Abel transform establishes a linear isomorphism between $C_{c}^{\infty}(\mathfrak{p})^{\mathcal{K}}$ and $C_{c}^{\infty}(\mathfrak{a})^{W}$. It is easily verified that $A$ : $C_{c}^{\infty}(\mathfrak{p})^{\mathcal{K}} \mapsto C_{c}^{\infty}(\mathfrak{a})^{W}$ is in fact a linear homeomorphism, so that the isomorphisms $A^{t}: \mathcal{D}^{\prime}(\mathfrak{a})^{W} \mapsto \mathcal{D}^{\prime}(\mathfrak{p})^{\mathcal{K}}$ and $\left(A^{-1}\right)^{t}: \mathcal{D}^{\prime}(\mathfrak{p})^{\mathcal{K}} \mapsto \mathcal{D}^{\prime}(\mathfrak{a})^{W}$ are defined.

Recall the symmetry $S$ from Section 4 , defined by $S f(x)=S f(-x)$, and note that, as maps from $C_{c}^{\infty}(\mathfrak{a})^{W}$ into itself, we have

$$
\begin{aligned}
D_{k} E_{0} & =D_{k} S D_{0}=(2 \pi)^{\frac{\operatorname{dim} \mathfrak{q}}{2}} D_{k} S \mathcal{F} A^{-1}=(2 \pi)^{\frac{\operatorname{dim} \mathfrak{q}}{2}} D_{k} S D_{k} \operatorname{Res}_{\mathfrak{a}}^{\mathfrak{p}} A^{-1} \\
& =(2 \pi)^{\frac{\operatorname{dim} \mathfrak{q}}{2}} D_{k} E_{k} \operatorname{Res}_{\mathfrak{a}}^{\mathfrak{p}} A^{-1}=(2 \pi)^{\frac{\operatorname{dim} \mathfrak{q}}{2}} \operatorname{Res}_{\mathfrak{a}}^{\mathfrak{p}} A^{-1} .
\end{aligned}
$$

Let $\operatorname{Ext}_{\mathfrak{a}}^{\mathfrak{p}}: C^{\infty}(\mathfrak{a})^{W} \mapsto C^{\infty}(\mathfrak{p})^{\mathcal{K}}$ be the inverse of $\operatorname{Res}_{\mathfrak{a}}^{\mathfrak{p}}$. Using (6.3) we find, for $f \in C^{\infty}(\mathfrak{a})^{W}$ and $\psi \in C_{c}^{\infty}(\mathfrak{a})^{W}$,

$$
\begin{aligned}
\left\langle W_{k} f, \psi\right\rangle_{\mathfrak{a}} & =(2 \pi)^{\frac{\operatorname{dim} \mathfrak{a}}{2}} c_{k}^{-1}\left\langle\left(D_{k} E_{0}\right)^{t}\left(f w_{k}\right), \psi\right\rangle_{\mathfrak{a}} \\
& =(2 \pi)^{\frac{\operatorname{dim} \mathfrak{a}}{2}} c_{k}^{-1}\left\langle f w_{k}, D_{k} E_{0} \psi\right\rangle_{\mathfrak{a}} \\
& =(2 \pi)^{\frac{\operatorname{dim} \mathfrak{p}}{2}} c_{k}^{-1}\left\langle f w_{k}, \operatorname{Res}_{\mathfrak{a}}^{\mathfrak{p}} A^{-1} \psi\right\rangle_{\mathfrak{a}} \\
& =\left\langle\operatorname{Ext}_{\mathfrak{a}}^{\mathfrak{p}} f, A^{-1} \psi\right\rangle_{\mathfrak{p}} \\
& =\left\langle\left(A^{-1}\right)^{t} \operatorname{Ext}_{\mathfrak{a}}^{\mathfrak{p}} f, \psi\right\rangle_{\mathfrak{a}} .
\end{aligned}
$$

We conclude that $W_{k}=\left(A^{-1}\right)^{t} \operatorname{Ext}_{\mathfrak{a}}^{\mathfrak{p}}$, as maps with domain $C^{\infty}(\mathfrak{a})^{W}$. For the right-hand side, we know a priori only that it maps $C^{\infty}(\mathfrak{a})^{W}$ into $\mathcal{D}^{\prime}(\mathfrak{a})^{W}$, but this equality and Theorem [5.1 show that it is actually a linear automorphism of $C^{\infty}(\mathfrak{a})^{W}$. It follows from this that $\left(A^{-1}\right)^{t}$, which a priori is only known to map $C^{\infty}(\mathfrak{p})^{\mathcal{K}} \subset \mathcal{D}^{\prime}(\mathfrak{p})^{\mathcal{K}}$ into $\mathcal{D}^{\prime}(\mathfrak{a})^{W}$, in fact establishes a linear isomorphism between $C^{\infty}(\mathfrak{p})^{\mathcal{K}}$ and $C^{\infty}(\mathfrak{a})^{W}$. This enables us to identify the restriction of Dunkl's intertwiner to $C^{\infty}(\mathfrak{a})^{W}$ as $V_{k}=\operatorname{Res}_{\mathfrak{a}}^{\mathfrak{p}} A^{t}$, where the fact that $A^{t}: C^{\infty}(\mathfrak{a})^{W} \mapsto C^{\infty}(\mathfrak{p})^{\mathcal{K}}$ is a linear isomorphism is then implicitly used in the interpretation of the right-hand side.

Remark 6.1. For certain multiplicities of the restricted roots, the results on shift operators can be used to essentially invert the Abel transform, as follows. Assume that $k_{\alpha}=1 / 4 \sum_{\beta \in \mathbb{R} \alpha \cap \Sigma} m_{\beta}$ is an integer for all $\alpha \in R$. Then (4.10) implies that there exists a $W$-invariant differential operator $\widetilde{D}$ of order $1 / 4 \sum_{\alpha \in \Sigma} m_{\alpha}$, with poles along the singular hyperplanes only, such that $E_{k} g=\widetilde{D} E_{0} g$ for all rapidly 
decreasing $g \in C^{\infty}(\mathfrak{a})^{W}$. Therefore, if $f \in C_{c}^{\infty}(\mathfrak{a})^{W}$, we find that $\operatorname{Res}_{\mathfrak{a}}^{\mathfrak{p}} A^{-1} f=$ $(2 \pi)^{-\frac{\operatorname{dim} q}{2}} D_{k} S D_{0} f=(2 \pi)^{-\frac{\operatorname{dim} q}{2}} E_{k} D_{0} f=(2 \pi)^{-\frac{\operatorname{dim} q}{2}} \widetilde{D} E_{0} D_{0} f=(2 \pi)^{-\frac{\operatorname{dim} q}{2}} \widetilde{D} f$. This shows that the Abel transform is for such multiplicities essentially inverted by the $W$-invariant differential operator $(2 \pi)^{-\frac{\operatorname{dim} q}{2}} \widetilde{D}$.

Remark 6.2. In this section it has become clear that the theory of spherical functions for the Cartan motion group is intimately connected with the invariant part of the theory of Dunkl operators. It can, however, not be excluded that the general theory of these operators is also relevant in this context, for conceptual reasons which have gone unnoticed sofar. The author at least finds it hard to believe that, e.g., the description of the algebra of radial parts should not be the reflection of some more direct connection. There are, in effect, two additional indications that such a connection may exist.

The first of these is given by the estimates on the spherical functions $\psi_{\lambda}$. One has the integral representation

$$
\psi_{\lambda}(x)=\int_{\mathcal{K}} e^{(\lambda, k x)} d k
$$

for $\lambda \in \mathfrak{a}_{\mathbb{C}}$ and $x \in \mathfrak{a}$. The Kostant convexity theorem [19, Theorem 10.2, p. 473] then yields the estimate $\left|\psi_{\lambda}(x)\right| \leq \exp \left(\max _{w \in W} \operatorname{Re}(w \lambda, x)\right)$. But alternatively, this also follows from the estimate for $\operatorname{Exp}_{W}$ in Section 2 and the identification of the spherical functions as generalized Bessel functions, as explained in this section. This natural occurrence of $\operatorname{Exp}_{W}$ points to relevance of the original Dunkl operators in the Cartan motion group case.

The second indication consists of Torossian's application of Dunkl operators in the inversion of the Chevalley restriction isomorphism between the $\mathcal{K}$-invariant polynomials on $\mathfrak{p}$ and the $W$-invariant polynomials on $\mathfrak{a}$ [38. Here the original Dunkl operators make an appearance, not just the operators corresponding to invariant polynomials.

Remark 6.3. When the results in this section are combined with similar results for the Cherednik operators of Section 4.3 and with Theorem 4.13, one obtains a limit transition from the spherical functions for a Riemannian symmetric space of the non-compact type to the spherical functions for the corresponding Cartan motion group. This has previously been proved in a direct fashion by Ben Saïd and Ørsted in 2. This limit transition enables one to obtain explicit formulas for the spherical functions for the Cartan motion group.

\section{REFERENCES}

1. M. Abramowitz and I.A. Stegun, eds., Handbook of mathematical functions, Dover Publications, New York, 1970. MR0167642 (29:4914) (review of the 1964 original edition)

2. S. Ben Saïd and B. Ørsted, Analysis on flat symmetric spaces, J. Math. Pures Appl. 84 (2005), 1393-1426. MR2170768

3. S. Ben Saïd and B. Ørsted, Bessel functions for root systems via the trigonometric setting, Int. Math. Res. Not. 2005 (9), 551-585. MR2131449 (2006d:33022)

4. I. Cherednik, A unification of Knizhnik-Zamolodchikov equations and Dunkl operators via affine Hecke algebras, Invent. Math. 106 (1991), 411-432. MR.1128220 (93b:17040)

5. J.F. van Diejen and L. Vinet, Calogero-Moser-Sutherland Models, CRM Series in Mathematical Physics, Springer, 2000. MR:1843558 (2002b:37003)

6. C.F. Dunkl, Reflection groups and orthogonal polynomials on the sphere, Math. Z. 197 (1988), 33-60. MR0917849 (89b:42016) 
7. C.F. Dunkl, Differential-difference operators associated to reflection groups, Trans. Amer. Math. Soc. 311, (1989), 167-183. MR0951883 (90k:33027)

8. C.F. Dunkl, Operators commuting with Coxeter group actions on polynomials, in "Invariant theory and tableaux," Minneapolis, 1988, IMA Vol. Math. Appl. 19, 107-177. MR1035491 (91g:20060)

9. C.F. Dunkl, Integral kernels with reflection group invariance, Canad. J. Math. 43 (1991), 1213-1227. MR 1145585 (93g:33012)

10. C.F. Dunkl, Hankel transforms associated to finite reflection groups, in "Proceedings of the special session on hypergeometric functions on domains of positivity, Jack polynomials and applications," Tampa, 1991, Contemp. Math. 138, 123-138. MR 1199124 (94g:33011)

11. C.F. Dunkl, Intertwining operators associated to the group $S_{3}$, Trans. Amer. Math. Soc. 347 (1995), 3347-3374. MR1316848 (97b:22009)

12. C.F. Dunkl, Singular polynomials for the symmetric groups, Int. Math. Res. Not. 2004 (67), 3607-3635. MR 2129695

13. C.F. Dunkl, Singular polynomials and modules for the symmetric groups, Int. Math. Res. Not. 2005 (39), 2409-2436. MR2181357

14. C.F. Dunkl, M.F.E. de Jeu and E.M. Opdam, Singular polynomials for finite reflection groups, Trans. Amer. Math. Soc. 346 (1994), 237-256. MR.1273532 (96b:33012)

15. C.F. Dunkl and E.M. Opdam, Dunkl operators for complex reflection groups, Proc. London Math. Soc. (3) 86 (2003), 70-108. MR.1971464(2004d:20040)

16. C.F. Dunkl and Y. Xu, Orthogonal polynomials of several variables, Cambridge Univ. Press, 2001. MR:1827871 (2002m:33001)

17. G.J. Heckman, A remark on the Dunkl differential-difference operators, in "Harmonic analysis on reductive groups," Brunswick, 1989, Progr. Math. 101, 181-191. MR.1168482 (94c:20075)

18. G.J. Heckman, Dunkl operators, Séminaire Bourbaki 828, 1996-97, Astérisque 245 (1997), 223-246. MR1627113 (2000i:33021)

19. S. Helgason, Groups and geometric analysis, Academic Press, New York, 1984. MR0754767 (86c:22017)

20. S. Helgason, Geometric analysis on symmetric spaces, Amer. Math. Soc., Providence, RI, 1994. MR 1280714 (96h:43009)

21. L. Hörmander, The analysis of linear partial differential operators I, Springer, 1983. MR0717035 (85g:35002a)

22. M.F.E. de Jeu, The Dunkl transform, Invent. Math. 113 (1993), 147-162. MR.1223227 (94m:22011)

23. M.F.E. de Jeu, Dunkl operators, Thesis, Leiden University, 1994.

24. M.F.E. de Jeu, Subspaces with equal closure, Constr. Approx. 20 (2004), 93-157. MR2025416 (2005e:41083)

25. G. Lusztig, Affine Hecke algebras and their graded version, J. Amer. Math. Soc. 2 (1989), 599-685. MR0991016 (90e:16049)

26. E.M. Opdam, Some applications of hypergeometric shift operators, Invent. Math. 98 (1989), 1-18. MR1010152 (91h:33024)

27. E.M. Opdam, Dunkl operators, Bessel functions and the discriminant of a finite Coxeter group, Comp. Math. 85 (1993), 333-373. MR.1214452 (95j:33044)

28. E.M. Opdam, Harmonic analysis for certain representations of graded Hecke algebras, Acta Math. 175 (1995), 75-121. MR1353018(98f:33025)

29. E.M. Opdam, Lecture notes on Dunkl operators for real and complex reflection groups, MSJ Memoirs 8, Math. Soc. of Japan, Tokyo, 2000. MR.1805058(2003c:33001)

30. M. Rösler, Positivity of Dunkl's intertwining operator, Duke Math. J. 98 (1999), 445-463. MR 1695797 (2000f:33013)

31. M. Rösler, Dunkl operators: theory and applications, in "Orthogonal polynomials and special functions," Leuven, 2002, Lecture Notes in Math. 1817, 93-135. MR2022853 (2004k:33024)

32. M. Rösler and M.F.E. de Jeu, Asymptotic analysis for the Dunkl kernel, J. Approx. Theory 119 (2002), 110-126. MR1934628 (2003j:33035)

33. M. Rösler and M. Voit, Markov processes related with Dunkl operators, Adv. Appl. Math. 21 (1998), 575-643. MR 1652182 (2000j:60019)

34. W. Rudin, Functional analysis, Tata McGraw-Hill, New Delhi, 1973. MR0365062 (51:1315)

35. L. Schwartz, Théorie des distributions, Hermann, Paris, 1978. MR0209834(35:730) 
36. S. Thangavelu and $\mathrm{Y} . \mathrm{Xu}$, Generalized translation and convolution operator for the Dunkl transform, J. Anal. Math. 97 (2005), 25-56.

37. E.C. Titchmarsh, The theory of functions, Oxford Univ. Press, 1986.

38. C. Torossian, Une application des opérateurs de Dunkl au théorème de restriction de Chevalley, C.R. Acad. Sci. Paris 318 (1994), 895-898. MR.1278147 (95h:22010)

39. K. Trimèche, The Dunkl intertwining operator on spaces of functions and distributions and integral representation of its dual, Integral Transform. Spec. Funct. 12 (2001), 349-374. MR.1872375 (2002m:33012)

40. K. Trimèche, Paley-Wiener theorems for the Dunkl transform and Dunkl translation operators, Integral Transform. Spec. Funct. 13 (2002), 17-38. MR1914125 (2004e:44006)

41. Y. Xu, Integration of the intertwining operator for h-harmonic polynomials associated to reflection groups, Proc. Amer. Math. Soc. 125 (1997), 2963-2973. MR1402890 (97m:33004)

Mathematical Institute, Leiden University, P.O. Box 9512, 2300 RA Leiden, The NetherlandS

E-mail address: mdejeu@math.leidenuniv.nl 